\title{
ASPETTI DEL DIBATTITO OTTOCENTESCO PER LA NUOVA FACCIATA DEL DUOMO
}

\author{
AMEDEO BELLINI (*)
}

RIASSUNTO. - Nel 1886 venne bandito un concorso internazionale per il progetto di una fronte del Duomo di Milano che diede luogo ad un vivace dibattito sui caratteri stilistici che essa avrebbe dovuto avere. Si contrappongono due gruppi: coloro che giudicano l'edificio opera gotica la cui architettura risente di influenze straniere e ritengono quindi che la nuova fronte dovrà ispirarsi alle grandi cattedrali francesi e tedesche, in particolare avere torri laterali; coloro che invece ritengono l'opera strutturalmente romanico lombardesca, con forme esteriori gotiche, e sostengono una fronte a capanna. Fra questi Luca Beltrami che interpreta e spiega le forme del Duomo utilizzando le teorie di Charles Darwin.

$* * *$

ABSTRACT. - In 1886 was announced the holding of an international competitive examination concerning the design of the front of the Cathedral in Milan, which aroused a lively debate over its stylistic characteristics.

Two groups were opposed: those who considered the building a Gothic architecture featuring foreign influences, thus believing that the new front would have to be inspired by the great French and German cathedrals - in particular it would have to have side towers; those, on the contrary, who considered the building structurally a Lombardy Romanesque one with Gothic exterior forms advocated a hut-shaped front. Among these there was Luca Beltrami, who interpreted and explained the Cathedral shapes by employing Charles Darwin's theories.

Volumi che si propongano di illustrare il Duomo di Milano ai cittadini ed ai forestieri si susseguono con regolarità durante la prima metà del secolo XIX. Se ne indagano le origini, con variabile senso cri-

(*) Istituto Lombardo Accademia di Scienze e Lettere. Politecnico Milano. 
tico; si discute della sua fondazione come opera voluta e patrocinata da Gian Galeazzo Visconti, Conte di Virtù (o Vertù), o al contrario sorta per volontà popolare, con qualche conseguenza anche sulla valutazione della rilevanza della presenza di artisti stranieri nel cantiere. Comunque se ne tessono le lodi, la magnificenza, la grandezza, e se talora si accenna all'esigenza del suo completamento, ciò riguarda elementi la cui forma generale non è in discussione, come è il caso dei gugliotti che si uniformano a quanto già esiste.

E' oltre la metà del secolo che si presenta con forza crescente il tema della fronte, questione centrale dal 1882 al primo decennio del novecento; si osserva, in genere con un giudizio negativo, la presenza di elementi architettonici che non rispettano lo stile generale del monumento ed altri che appaiono invece di qualità, formali e tecnico-costruttive, troppo modeste per rimanere in opera.

In effetti il Duomo poteva considerasi opera finita, o quanto meno fabbrica per la quale non occorrevano interventi il cui progetto richiedesse scelte di fondo, con il completamento della facciata iniziato nel 1809 a seguito di un imperioso editto di Napoleone Bonaparte e concluso nel 1813. Quella che si manifesta a fine secolo è dunque una volontà di compiutezza ideale che supera gli aspetti funzionali, riguarda prevalentemente l'edificio in se stesso, come oggetto che deve presentarsi senza contraddizioni, e tale appariva stilisticamente la fronte napoleonica, per il suo valore simbolico e rappresentativo della cultura architettonica della città. Si registra anche un dibattito intorno alla sistemazione della piazza del Duomo, molto vivace, che riguarda questioni di forma generale dello spazio, di funzionalità a fronte delle esigenze del traffico, aspetti di natura architettonica; si discute specialmente del possibile prolungamento dei partiti architettonici mengoniani, non mancano considerazioni sulla fronte in rapporto all'ampiezza della piazza ed alle nuove visuali determinate dal variare degli assetti urbanistici con l'apertura di nuovi sbocchi stradali, ma tutto ciò non conduce mai ad indicazioni stringenti per quanto riguarda le scelte fondamentali (Fig. 1).

In realtà l'insoddisfazione per la situazione della fronte del Duomo assume una particolare dimensione quando intervengono considerazioni che muovendo proprio dalla constatazione della sua compiutezza ne propongono il restauro per giungere all'integrità di stile con una fronte adeguata all'organismo complessivo: la ricerca nella storia del monumento dei fondamenti teorici e tecnici per la progettazione di questa parte essenziale dell'organismo ne è la prova più evidente. Gli studi storici sull'edificio sono finalizzati alla ricerca dei dati che possano determinare con 
certezza, per taluni scientifica, le forme da dare alla nuova fronte. Interrogare i documenti sull'alterna presenza nel cantiere del Duomo degli architetti italiani o stranieri assume un significato speciale per definirne lo stile fondamentale: il gotico o un lombardesco nella sostanza strutturale e distributiva che circostanze e moda conducono a travestirsi con forme estranee alla cultura italiana, per citare la principale controversia. Entrano in gioco questioni di grande rilevanza: la funzione della storia per la conoscenza e le decisioni del momento attuale; il concetto di stile, dei modi della sua formazione e della sua analisi; la contrapposizione tra la realtà del monumento come documento della storia e manifestazione di un sentimento d'arte, il suo essere testimonianza di un'epoca esclusiva o prevalente, o piuttosto di un trascorrere del tempo in cui ogni momento lascia un segno. Sono i problemi che hanno caratterizzato la questione del restauro, presenti in una dimensione che ne documenta il nesso profondo con i temi della progettazione architettonica, non soltanto per l'identità delle forme espressive che l'architetto utilizza, ma soprattutto per la solida coscienza di agire in un'epoca di eclettismo, fatto che assume il significato, specialmente in Boito, di una condizione di privilegio, che assicura all'epoca presente capacità di giudizio, di scelta non condizionata da una teoria, da un punto di vista esclusivo. Tutto ciò si intreccia nella contrapposizione tra chi tende a far prevalere comunque una teoria dell'architettura, per esempio sostenendo la prevalenza assoluta degli aspetti tecnici, la necessità di un esame razionale e chi tende invece a valutare le qualità espressive, il che comporta anche l'esame estremamente analitico dell'architettura o la ricerca di una sintesi che non scaturisce affatto dall'esame di singoli elementi. L'intreccio delle posizioni è complesso, perché, come accade con una certa frequenza nel dibattito sul restauro, l'identità o la vicinanza delle posizioni teoriche non conducono necessariamente ad una comunanza di posizioni pratiche.

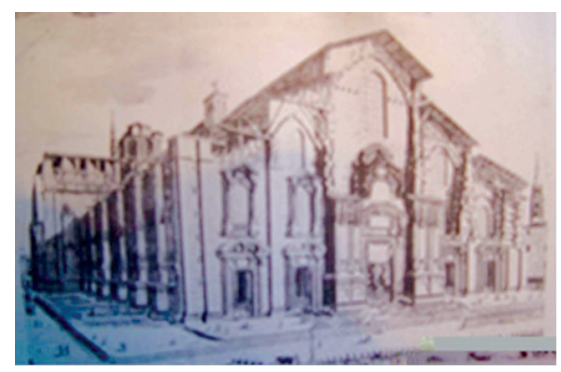

Fig. 1. Il Duomo prima della costruzione della fronte in epoca napoleonica, dal volume di Camillo Boito, Il Duomo di Milano, Milano 1889, tavola 43. 
Sarebbe vano esaminare il dibattito nel suo susseguirsi cronologico, per la sua ripetitività, quanto meno nei percorsi analitici; più utile invece constatare le diverse posizioni, avvertendo di volta in volta i loro limiti, dovuti alle evidenti strumentalizzazioni del resoconto storiografico, piegate alle esigenze dimostrative dell'autore. Le ricerche attorno al Duomo sono un esempio, se mai ce ne fosse bisogno, della relatività della storiografia alle intenzioni dell'autore.

La pubblicazione dei documenti della fabbriceria ${ }^{1}$ costituisce un punto di riferimento inevitabile per tutte le opere successive: con essi si rendono noti i documenti tecnici e contabili, i rapporti tra l'amministrazione e gli architetti di volta in volta interpellati per la prosecuzione della fabbrica; la loro presenza determina l'abbandono dei riferimenti storiografici più antichi in quelle parti che riferiscono di relazioni scomparse, di quelli che si fondano anche sulla tradizione orale non comprovata, ${ }^{2}$ che tuttavia troverà qualche estimatore, come vedremo (Fig. 2).

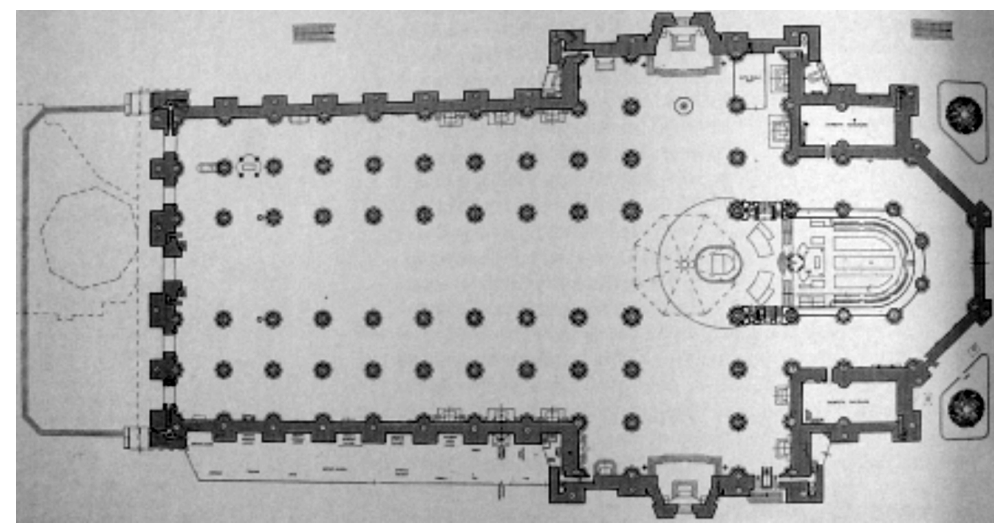

Fig. 2. Pianta del Duomo di Milano, disegno moderno della fabbriceria in occasione dei recenti restauri statici.

1 Annali della fabbrica del Duomo di Milano dall'origine fino al presente, pubblicati per cura della sua amministrazione, Milano 1877-1885, prima libreria editrice G. Brigola, poi E. Reggiani e C.; sei volumi con una prefazione e note illustrative di Cesare Cantù.

2 Una Bibliografia ragionata, molto estesa, è pubblicata da F. Salveraglio, in C. Boito. Il Duomo di Milano e i disegni per la sua facciata, tipografia Luigi Marchi, Milano 1889. 
Una quindicina di anni prima era stata pubblicata una illustrazione del Duomo corredata da una imponente documentazione grafica, piante prospetti, sezioni, particolari costruttivi e decorativi, che sarà di grande utilità per gli studi sulla nuova fronte; il volume comprende anche testi storico-descrittivi che non offrono però particolari elementi di novità (Fig. 3). ${ }^{3}$

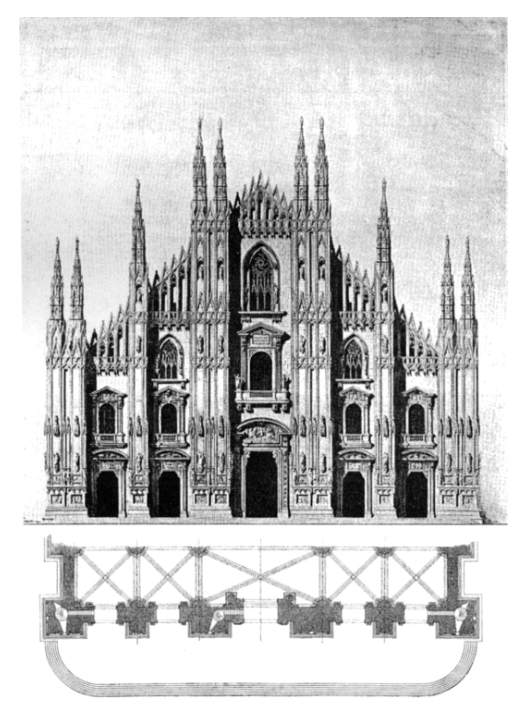

Fig. 3. Fronte del Duomo di Milano, dal volume di Fermo Zuccari, Giovanni De Castro, Giuseppe ed Antonio Bramati, Il Duomo di Milano rappresentato in sessanta tavole ed illustrato da cenni storici e descrittivi, Milano 1863, figura 5.

3 F. Zuccari, G. De Castro, G. e A. Bramati, Il Duomo di Milano rappresentato in sessanta tavole illustrate da cenni storici e descrittivi, P. e G. Vallardi, Milano 1863; De Castro e l'architetto Zuccari, il Magister comacinus che firma vari articoli sul Giornale dell'ingegnere architetto e agronomo, sono autori degli scritti storico descrittivi; ai fratelli Bramati, noti incisori, fra di loro indistinguibili quando non siglano le proprie tavole, si devono le belle tavole. Il volume ha una seconda edizione nel 1871, ed una terza, la prima in francese, nel 1881. Una più recente riproduzione è contenuta in Il Duomo di Milano, Editalia, Roma 1996 in una veste editoriale lussuosa e pomposamente volgare nella quale sono aggiunti testi moderni, in forma antologica, privi del minimo apparato orientativo e dei necessari riferimenti esplicativi: un contesto che svilisce gli ottimi saggi di autori contemporanei (G.B. Sannazzaro. L'architettura; M. Di Giovanni Madruzza. Le decorazioni; E. Brivio. Le facciate; R. Bernabei. La questione della facciata). 
Una prima presa di posizione appare in due articoli, uno di Tito Vespasiano Paravicini ${ }^{4}$, l'altro di Paolo Cesa-Bianchi, ${ }^{5}$ pubblicati contemporaneamente, a cui risponde Camillo Boito. ${ }^{6}$ Paravicini, che aveva aderito alle tesi ruskiniane con minori equivoci di quanto si riscontri presso i pochi italiani che erano iscritti alla S.P.A.B., esprime la necessità della ricerca dei fatti, di una storiografia che rifiuti tesi incontrollate, schemi ritenuti indiscutibili, che si riferisca a modelli ed archetipi, ma si basi piuttosto su migliori classificazioni dei monumenti, più articolate; svolgendo argomentazioni storiografiche e critiche egli sostiene l'esistenza di uno "stile lombardo", che egli chiama anche "gotico anteriore", da cui il Duomo deriva soprattutto per quanto concerne gli aspetti strutturali, profondamente diverso da quello d'oltralpe. Il contrasto tra gli architetti italiani e quelli stranieri è proposto come un vero e proprio scontro di scuole mentre il successivo continuo appello ad artefici d'oltralpe, la cui presenza è comunque per ciascuno di essi poco duratura, come un tentativo da parte del Duca Gian Galeazzo e poi della fabbriceria, di avere una sorta di arbitrato nei contrasti tra "le chiesuole ... le amicizie e le inimicizie artistiche che si sogliono creare e le gare di corpo e di paese". Egli tende ad escludere che il problema fosse di natura puramente statica, perché in caso contrario si sarebbe risolto attraverso il calcolo ${ }^{7}$ mentre le questioni dimensionali e di forma dei pilastri sono ricondotti a questioni di scuola. La presenza o l'as-

4 T.V. Paravicini, Considerazioni sul Duomo di Milano, in Atti del Collegio degli Ingegneri ed architetti in Milano, Tipografia degli ingegneri, Milano 1878, pp. 218-224, ma anche in Il Politecnico, anno XXVII, Milano 1879, pp. 38-44 e in opuscolo autonomo.

5 P. Cesa-Bianchi, La monumentalità del Duomo di Milano dimostrata dalla sua essenza artistica, dalla continuità dei lavori richiesti per la conservazione del medesimo, e dalla necessità d'opere pei contingibili restauri, e delle altre non d'innovazione ma del graduale compimento del tempio, in Atti del Collegio degli Ingegneri ed architetti in Milano, Milano 1878, pp. 207-208, una sintesi in Il Politecnico, anno XXVII, Milano 1879, pp.187-188, nella rubrica dedicata agli Atti del Collegio..., ove si riferisce della seduta del giorno 24 novembre 1878 .

6 C. Boito, Osservazioni sulla conservazione, sui restauri, e sulla Amministrazione del Duomo di Milano a seguito della discussione iniziata con le letture di T.V. Paravicini e P. Cesa-Bianchi, in Atti del Collegio degli Ingegneri ed architetti in Milano, Milano 1878 , pp. 210-212.

7 L'affermazione mette in luce una certa ingenuità dell'autore perché da un lato egli sembra ignorare l'assenza reale di metodi di calcolo all'epoca della costruzione del Duomo, dall'altro il fatto che una controversia sul dimensionamento era effettivamente intervenuta tra chi lo impostava sul triangolo equilatero e chi lo voleva diretto da proporzioni regolate dal triangolo egizio. 
senza di riseghe nei contrafforti, il loro andamento a parallelepipedo o piramidale, la loro direzione, specie nelle zone absidali, radiale o perpendicolare rispetto alla forma dei piloni, lo sviluppo parziale o per tutta l'altezza dei muri perimetrali, sono considerate differenze essenziali. Al contrario gli aspetti puramente formali, la geometria basata sul triangolo, la presenza di cuspidi o pinnacoli, gli appaiono irrilevanti: si tratta di elementi architettonici presenti in stili anteriori, anche orientali, specialmente asiatici, e quindi non individuanti l'originalità artistica. ${ }^{8} \mathrm{Il}$ completamento, la fronte soprattutto, dovrà quindi ispirarsi alla tradizione italiana e non prevedere, per esempio, campanili.

Paolo Cesa-Bianchi in una lunga memoria riprende le vicende storiche del Duomo, sottolinea quanto si debba fare per ordinaria o straordinaria manutenzione, dedica una parte della sua relazione alle opere necessarie al completamento dell'edificio che siano del tutto nuove: la fronte, il pavimento, le porte, la decorazione delle volte. Per quanto riguarda la facciata egli afferma l'esigenza di mantenere una conformità di stile con l'edifico, che considera gotico secondo progetti di origine estera. Gli appare necessario quindi la presenza dei campanili che $\mathrm{Pa}$ ravicini aveva escluso.

La risposta di Boito dissente dai precedenti, ripercorre la storia dell'edificio sostenendo la probabile origine francese del primo progetto, tenendo conto anche delle relazioni con la Francia di Gian Galeazzo, del suo titolo di Conte di Vertu nella "Sciampagna", sottolinea la presenza di cattedrali archiacute in quella regione (Troyes, Chalon, Reims) mentre poco lontano si trova quella di Bouvais che presenta analogie con la cattedrale di Milano e quella di Bologna. Ancora: la presenza di architetti stranieri non può che determinare una influenza; la stessa presenza di una guglia centrale non può essere prova di un'origine lombarda: ne sussistono esempi in area renana e normanna. Boito fa riferimento anche alle particolarità costruttive per rilevare la presenza di riseghe anche nel Duomo, sia pure poco accentuate e soprattutto lo sviluppo dei pinnacoli che ha il suo riscontro nella cattedrale di Colonia.?

8 Si noti che le valutazioni critiche espresse da Paravicini sull'esito della seconda fase del progetto internazionale hanno un carattere formale che tiene assai poco conto di questa impostazione, cfr. A. Bellini. Tito Vespasiano Paravicini, Guerini, Milano 2000, p.138, nota 10 .

9 Le tesi appaiono quindi molto vicine a quelle di Cesa-Bianchi, con cui però sorge una vivace polemica in relazione ai lavori in corso che egli critica vivacemente, in 
Questa breve ma intensa discussione anticipa i temi che staranno alla base di quella futura per la realizzazione della nuova facciata, che muoveranno concretamente dal concorso bandito dall'Accademia braidense nel 1883, non esclusi i problemi di rapporto tra fabbriceria e stato sul piano finanziario e soprattutto delle reciproche competenze nelle scelte di natura prettamente artistica, e quindi dei rapporti con la Commissione conservatrice dei monumenti e il Consiglio superiore di antichità e belle arti. ${ }^{10}$

Un'analisi fondata sulle valutazioni tecnico-costruttive, sulla razionalità intrinseca dell'organismo, sulla definizione della natura dello stile del Duomo e nello stesso tempo la dimostrazione che tutto ciò non esaurisce i termini della questione della fronte sono argomento di numerosi scritti di Luca Beltrami, ${ }^{11}$ frutto di studi preliminari alla parteci-

particolare per la collocazione in opera delle statue colossali di Mosè e di Aronne, che giudica erroneamente proporzionate.

10 Boito vorrebbe sottrarre alla fabbriceria ogni competenza di natura artistica e quindi propone un ordine del giorno nel quale si richiede, fra altro, la costituzione di un organo consultivo competente in questioni d'arte, con l'obbligo per la fabbriceria di valersene; la proposta verrà attenuata e si approverà un documento in cui l'obbligo si trasforma in raccomandazione. Anche in futuro non mancheranno attriti nei rapporti tra fabbriceria e organi di controllo ministeriale, non senza ambiguità soprattutto dopo l'esito del secondo concorso internazionale, quando il ministero rinuncia a dare un giudizio sulla eseguibilità del progetto vincitore, per sottrarsi alle polemiche sorte attorno all'esito del concorso.

11 Sono 47 le pubblicazioni dedicate al Duomo da Luca Beltrami, frutto sia di ricerche archivistiche che portano alla conoscenza di importanti documenti e disegni inediti, sia di riflessione critica. Fra le principali: La facciata del nostro Duomo, Colombo e Cordani, Milano 1883; Per la facciata del Duomo di Milano, Colombo e Cordani, Milano 1887; Elementi decorativi ed architettonici, componenti la facciata del Duomo di Milano, secondo il progetto dell'arch. Luca Beltrami, Colombo e Cordani, Milano 1888; Francesco Maria Richino autore di un progetto per la facciata del Duomo, rimasto sconosciuto, in Archivio storico Lombardo, anno XV, 1888, pp, 670-677; Per la costruzione del Duomo di Milano. Disegni inediti del 1390; Il modello per la nuova facciata del Duomo di Milano e il disegno della Torre campanaria, dell'arch. Luca Beltrami, Demarchi, Milano 1892; Sul restauro al rivestimento dei piloni nel Duomo di Milano, tipografia Bernardoni, Milano 1893; Pro Domo, Allegretti, Milano 1899; Il coronamento del Duomo di Milano in base ad antichi disegni in parte inediti, Allegretti, Milano 1900; La porta settentrionale del Duomo di Milano: vicende e raffronti, Allegretti, Milano 1900; La tutela artistica del Duomo di Milano nell'ultimo quarto del secolo XIX, Pagnoni, Milano 1900; Le guglie del Duomo di Milano, in Rassegna d'Arte, n. ${ }^{\circ}$ 1, Milano 1901; Il tiburio del Duomo. Nuove indagini e nuovi documenti, in La Perseveranza, Milano 15 novembre, 6 e 15 dicembre 1902; Leonardo da Vinci negli studi della facciata per il tiburio della Cattedrale di Milano, Allegretti, Milano 1903. Per un elenco completo vedi: Bibliografia degli scritti di Luca Beltrami, Allegretti, Milano 1930. 
pazione ai tre concorsi di progettazione, sia successivi al loro esito, per lui sfortunato, nei quali occorre spesso distinguere attentamente le convinzioni dello studioso dalle recriminazioni personali, dalle puntigliose difese delle proprie scelte artistiche. ${ }^{12}$

Le proposizioni di base sono quelle che affermano la necessità di muovere da una valutazione di natura storica, ma che sarebbe erroneo considerare soltanto questioni di stile, soprattutto se esse vengono intese in termini puramente formali, osservando ciò che si vede nel monumento o in altri "coevi e congeneri"; occorre anche l'attenta considerazione di ragioni economiche, e quindi la verifica della possibilità di costruzione, l'analisi della struttura topografica del suo ambiente, delle condizioni di viabilità.

Egli definisce l'edificio di "stile ibrido", e con questo non afferma soltanto che vi appaiono elementi formali contrastanti, accoppiamenti di stile, ma anche che vi sono incoerenze organiche, in particolare indica come errori i contrafforti d'angolo trigemini, quelli centrali che sono raddoppiati e non corrispondono a qualsiasi struttura interna, rendendo misera la parte centrale della fronte che non ha sufficiente sviluppo orizzontale, pur essendo troppo bassa.

Già da questa impostazione si possono leggere: una critica al restauro di quegli anni, in cui il recupero dell'unità stilistica del monumento è spesso il ritorno ad una generica storicità, ad un ricollocare il monumento nell'epoca storica della sua origine assumendo come modello l'imitazione delle opere "coeve e congeneri", senza una valutazione della specifica realtà dell'edificio; una certa adesione alle proposizioni di Viollet-Le-Duc sul valore d'arte come presenza di una coerenza organica e decorativa senza incongruenze.

Beltrami esamina quindi le possibilità di intervento: introdurre soltanto elementi decorativi non farebbe altro che aggiungere ulteriori incongruenze, occorre dunque pensare ad un'opera più radicale. In

12 Si considerino, ad esemplificazione, alcuni fatti legati al concorso indetto dall'Accademia di Brera nel 1882: la commissione di giudizio aveva assegnato il primo premio ex aequo a Luca Beltrami ed a Carlo Ferrario, ma il consiglio accademico non aveva accettato il verdetto ed assegnato a quest'ultimo il primo premio; il giudizio verso Beltrami è sostanzialmente elogiativo ma ovviamente contiene alcune critiche, se non altro per giustificare la preferenza per il progetto di Ferrario, ma egli ne riferisce come di una valutazione superficiale e sostanzialmente sprezzante, $\mathrm{cfr}$. L. Beltrami. La facciata del nostro Duomo, Colombo e Cordani, Milano 1883, p.6. 
prima istanza egli esamina la questione che era apparsa determinante nel dibattito che lo aveva preceduto: costruire campanili laterali che occupino parte della fronte. A favore di questa tesi sta il fatto che essi sono presenti nell'architettura gotica, null'altro; a sfavore il fatto che, da un punto di vista organico il Duomo non ha nulla a che vedere con il gotico: è struttura semplice, specialmente nei piloni, non ha cappelle sporgenti, è privo della varietà di contrafforti che caratterizza quello stile, ha un basso rapporto tra area dei sostegni ed area coperta. Da un punto di vista estetico l'inserimento di torri peggiorerebbe il rapporto tra linee orizzontali e verticali a meno che esse avessero uno sviluppo pari a quello della guglia maggiore, eliminandosi così quel carattere di predominanza dell'incontro del piedicroce, le cui forme e il cui sviluppo sono caratteristica unica del Duomo.

Da queste osservazioni nascono le caratteristiche del progetto: correggere le proporzioni troppo depresse; dare maggior rilievo alla parte centrale della fronte; dare alle sue parti laterali un collegamento con il fianco; collocare un contrafforte laterale unico, massiccio, come nelle testate del capocroce; risvoltare sulla fronte la cimasa che corre sui fianchi; risvoltare il basamento; fare le finestre minori uguali a quelle laterali ma di decorazione più semplice per evitare una prevalenza della statuaria rispetto alla struttura architettonica (possibile l'uso dei filateri a spirale a lembo fiorito, come in alcune testate dei bracci); prevedere soltanto tre porte, soluzione che ha ambigui precedenti storici, perché le cinque porte eliminerebbero la continuità del basamento, determinano difficoltà compositive nella linea orizzontale costituita dalle incorniciature della porta maggiore. Per le porte egli raccomanda una decorazione molto intensa, non gotica, suggerisce l'uso della doratura, presente anche in altre parti del monumento, vuole la presenza di statue nella porta centrale.

In sintesi: rispetto dell'organismo interno, ricorrenze all'esterno; ricerca dei motivi nel Duomo stesso o in organismi di "consimile carattere"; economia, nella costruzione in aderenza alle condizioni economiche della fabbriceria e della città, escludendo tuttavia opere troppo limitate, ma anche riforme estreme, per l'esigenza di un fondamentale rispetto del monumento, per la continuità con la tradizione. ${ }^{13} \mathrm{E}$ non vi è chi non veda in questo il Beltrami conservatore, politicamente, che vede

13 La facciata del nostro Duomo, Colombo e Cordani, Milano 1883. 
il progresso soltanto nella lenta stratificazione, nell'accumulazione delle conoscenze, nella continuità sinergica delle opere.

Questi i principi, esposti nella relazione che accompagnava il progetto per il concorso accademico del 1883, ribaditi successivamente in quelli di preparazione del progetto per il concorso internazionale bandito nel $1886 .{ }^{14}$ Di particolare interesse ai nostri fini il secondo di questi ultimi, che contiene analisi e conclusioni che fondano il progetto. Beltrami non ritiene l'edificio definibile in astratto, come materializzazione di una serie di principi, ma piuttosto che esso sia il risultato di un "processo sperimentale": la mente osserva la preponderanza di certi caratteri decorativi e costruttivi, stabilisce istintivamente alcune categorie, i connotati dello stile; il procedimento è assimilato a quello di sistematizzazione delle scienze naturali, ma, in tutti e due i casi le teorie sono sempre più accentuate di quanto non esiga la realtà delle cose: "il bisogno di classificare e generalizzare per fare entrare in un sistema ci porta ad ammettere tipi astratti, ideali teorici, ai quali ci forziamo di adattare qualsiasi manifestazione per quanto originale o indispensabile".

Questa interessante posizione, in cui teoria e razionalità si fondono concretamente nella realtà sperimentale, è sostenuta da un richiamo a Taine che ebbe a lamentarsi di una educazione volta all'analisi minuziosa, che guarda alle immagini, alle classificazioni a scapito della realtà: prima la carta del terreno, egli dice, prima la nomenclatura (o gli esemplari in museo) degli animali; ciò determina che le manifestazioni vitali della società si fossilizzino nelle statistiche. Il concetto è ricondotto sull'architettura: non la teoria, ma l'analisi minuta e concreta direttamente sul monumento, considerato nella sua specifica individualità.

Tuttavia Beltrami, che caratterialmente tende a dare una spiegazione razionale a tutto ciò che lo circonda, sente il bisogno di dare unitarietà e finalizzazione all'osservazione sperimentale, ed egli la trova nella tesi che costituisce la sintesi della cultura positivista e scientifizzante: "Nessuno ha tentato di applicare alle continue variazioni di forme architettoniche il principio di Darwin della selezione naturale delle forme, oggi "principio degli adattamenti".

14 Per la facciata del Duomo di Milano, Colombo e Cordani, Milano 1887. Sotto questo titolo tre saggi: il primo Le linee fondamentali coincide con la pubblicazione del 1883; il secondo Lo stile dedicato alle caratteristiche organiche e formali degli edifici, in generale ed in riferimento al Duomo; il terzo, intitolato La teoria, si occupa soprattutto del proporzionamento geometrico della fabbrica. 
Gli stili, egli afferma, non hanno origine perfettamente definita e precisata per epoca e per località, la natura non fa salti, e Darwin ha sostenuto la tesi che le variazioni sono la manifestazione del continuo perfezionamento di ogni essere organico per mantenerlo in rapporto con le condizioni in cui vive: "Darwin ha dimostrato come la varietà, anche lieve di un individuo - trasmessa per generazioni e favorita nello sviluppo delle condizioni locali - si fissi, si completi, diventi razza". Così egli ritiene che avvenga per l'architettura: in essa si manifestano variazioni di poca entità, che rimangono casuali; variazioni in rapporto diretto con l'ambiente, che si riproducono, si accentuano, servono di base per forme affini, determinano uno stile; lo si verifica a grandi periodi "quando l'accumulo produce l'impronta speciale di un'epoca".

Le tesi che ponevano l'arte come diretta e sintetica manifestazione di una realtà sociale, note ed accolte in Italia attraverso gli insegnamenti e gli scritti di Pietro Selvatico ${ }^{15}$ ha qui una originale interpretazione, che tuttavia trascrive analiticamente una tesi più generale sulla nascita ed il significato dell'arte espressa da Tito Vignoli. ${ }^{16}$

Scrive Beltrami: "Io prevedo che a molti sembrerà strano principio materialista per cui lo stile architettonico - il quale si presenta es-

15 Soprattutto con l'opera Storia estetico-critica delle Arti del Disegno..., Pietro Naratovich, Venezia 1852-1856.

16 Tito Vignoli, presidente del Circolo Filologico di Milano, sede nella quale Beltrami espone in una conferenza le tesi sul Duomo in seguito pubblicate e qui esposte, è personaggio oggi poco noto ma che ebbe un ruolo di rilievo nella Milano di fine secolo. La sua opera è interessante soprattutto per una originale interpretazione del pensiero di Darwin, per la sua applicazione negli studi di antropologia e di psicologia. Egli sviluppa in modo particolare l'ipotesi di una continuità di sviluppo da una indistinta materia originaria all'uomo, considerando le forme di vita animale stati intermedi tuttora presenti, deducendone quindi la possibilità di comprendere la complessa psicologia umana anche attraverso lo studio del loro comportamento. Lo stesso processo esplicativo è applicato alle forme di espressione intellettuale, come l'arte. Vignoli non dubita della fisicità della forza che determina l'evoluzione, che in lui coincide con il progresso, ed ha fiducia che in futuro essa sia definita e descritta, come era avvenuto in un passato relativamente recente per l'energia elettrica. Gli scritti che possono meglio illustrare questi aspetti del suo pensiero sono: Saggio di una dottrina razionale del progresso, Vallardi, Milano 1863; Dell' $i$ stinto e dell' intelligenza, in Rendiconti del Regio Istituto Lombardo di Scienze e Lettere, serie 2a, vol. 2, Milano 1869, pp. 419-453; Della legge fondamentale dell'intelligenza nel regno animale, Dumolard, Milano 1877; Mito e scienza, Dumolard, Milano 1879; I tre fattori naturali dell'estetica, ricerca scientifica, in Rendiconti del Regio Istituto Lombardo di Scienze e Lettere, serie 2a, vol. 14, Milano 1881, pp. 426-447. 
senzialmente come concezione ed estrinsecazione affatto ideale del pensiero umano - è in realtà governato nel suo svolgimento da quella stessa legge che governa la natura: sembrerà strano il dover ammettere che le trasformazioni delle forme architettoniche siano sottratte alla diretta influenza della mente umana, per essere affidate ad una legge naturale di selezione. Ma a quel modo che le leggi scientifiche, coll'ammettere il principio di affinità elettive, o le leggi di attrazione e di gravitazione hanno dovuto piegarsi quasi a riconoscere un principio di vita nel mondo inorganico, così dobbiamo concedere che queste leggi abbiano, per converso, a varcare quel confine che noi le tracciamo per portare, dirò così, una influenza insciente in quel campo d'azione che vogliamo esclusivamente riservato alla mente umana". Ed anche: "Dovendo ora spiegare il fondamento di questa teoria della selezione naturale, estesa ed applicata alle evoluzioni delle forme architettoniche più ideali, non avrò che a mostrare come alcune di queste evoluzioni, siano la conseguenza delle leggi generali darwiniane. Una di queste leggi stabilisce che le specie indigene sono facilmente vinte dalle specie naturalizzate le quali emigrando, e cioè staccandosi dalle forme-madri, si adattano facilmente a subire le modificazioni del nuovo ambiente in cui si trovano.

L'esemplificazione tende a parallelismi molto stretti: se Darwin afferma che le specie indigene sono facilmente vinte dalle specie naturalizzate, ciò è tradotto nelle "cosiddette influenze", esemplificando non a caso con l'arco acuto che "nasce in oriente ove se ne trovano molti esempi sporadici, ma che non si sviluppa, non modifica l'organismo, si atrofizza, è variante inefficace dell'arco a tutto sesto, in occidente invece diviene fatto statico e coinvolge l'organismo. L'arte come la natura è prodiga di varietà ma avara di innovazioni e ciò che nella storia naturale è rappresentato dalle forme intermedie tra uno stato e l'altro, ha il suo corrispettivo in ciò che nell'arte sono le forme di transizione". ${ }^{17}$

Ancora Beltrami: "Rimane ad accennare a quelle circostanze le quali abbiano potuto aiutare queste forme occidentali nel formare uno stile: per questo mi riporto nuovamente alla teoria scientifica accennata.

17 Ulteriori esempi di questa analisi: il gotico è caratterizzato da volte leggere che invece il romano ha pesanti e rigide ragion per cui le costolonature, che ove sono presenti, non si sviluppano: prevale di principio di una staticità affidata al peso; il lombardesco è intermedio e attua un principio di distribuzione delle spinte che modifica la forma dei pilastri, esso è quindi base del gotico. 
La selezione naturale è particolarmente basata su quel principio di concorrenza vitale che Darwin, con frase evidente, definì: la lotta per l'esistenza. Ebbene, ogni forma architettonica è soggetta a questa legge: non è libera fantasia dell'uomo che ne governa le modificazioni, sibbene le esigenze dell'ambiente in cui queste forme si svolgono: le principali di tali esigenze sono quelle risultanti dai materiali di fabbrica, dalle condizioni di clima, dai criteri economici della regione nella quale l'edificio si svolge: l'intervento diretto della mente umana è secondario, e si riduce, si può dire, a prendere atto delle trasformazioni che subisce la forma, per improntarle del soffio dell'arte. Questo concetto delle modificazioni caratteristiche cui le forme architettoniche, coll'estendersi a regioni disparate, vanno soggette secondo i bisogni e le abitudini locali, venne per verità abbozzato molti anni or sono dall'Hope, ma nell'opera sua rimase in embrione, soffocato dalla teorie delle influenze per la quale l'Hope si lasciò trascinare a conclusioni alquanto fantastiche: io mi proverrò a svilupparle brevemente costatando, con qualche esempio, l'immediata e diretta azione di quelle condizioni locali sull'organismo architettonico, fermandomi in particolar modo sull'azione esercitata dai materiali costrutti, perché è quella che nel Duomo, come vedremo fra poco ebbe un'importanza capitale."

Beltrami afferma in sostanza che la teoria delle influenze non ha senso se interpretata in forma ideale la legge naturale della selezione delle forme, e la funzione prevalente dei materiali e del loro uso.

Ribadita la necessità di una osservazione diretta ed il rifiuto di una erudizione astratta, l'esame del Duomo lo conduce ad affermare che esso, centro della città, il luogo più importante della vita religiosa, ma anche civile, intellettuale, non avrebbe potuto abbandonare la tradizione locale, soprattutto in un'epoca nella quale le trasformazioni erano molto lente. Essa è rappresentata, nell'architettura religiosa, dal tipo della basilica lombarda, che si presenta storicamente secondo due forme: quella a colonne e quella a volte che dalla prima deriva. Il Duomo non appare strettamente derivato dall'una o dall'altra ma più simile alla seconda da cui differisce per il maggior numero di navate, per lo sviluppo di quelle minori attorno all'altare, per avere la sagrestia all'interno dell'organismo e per la forma particolare dell'abside.

Per ciascuna di esse Beltrami individua le cause: le prime due gli appaiono "estrinsecazione materiale di una volontà politica di magnificenza", in cui la scelta primaria è la seconda, mentre la prima sarebbe una conseguenza che ha un precedente in Santo Stefano a Verona.

Per quanto riguarda la forma absidale, ciò che molti considerano 
di provenienza nordica, essa sarebbe in realtà l'esito di una semplificazione del perimetro, divenuta insostenibile la volta sferica per la presenza delle cappelle attorno all'altare; nessun goticismo dunque. Per quanto riguarda la posizione della sacrestia si tratta di un ritorno all'antico, ad uno schema distributivo soppresso quando si erano introdotte le absidi minori.

La lettura delle vicende storiche conferma, ma sarebbe meglio dire deriva da queste premesse: la distribuzione e la struttura fondamentale sono lombardesche, ecco perché gli stranieri la criticano, non credono nella sua stabilità, non la comprendono; la massa decorativa dà un'impressione gotica, ma nei singoli elementi il Duomo è sviluppo di elementi lombardi, che Beltrami interpreta essenzialmente come variazioni in gran parte derivate dal passaggio dell'uso del cotto a quello del marmo: in certi casi si possono riprodurre motivi decorativi, per esempio gli archetti; in altri casi gli artefici si ispirano alle soluzioni già attuate laddove la pietra era già in uso: scelta razionale, non influenza estera.

Analiticamente Beltrami indica il basamento, di forme romanicolombarde tipiche; le pareti che non mostrano alcun reale cambiamento per l'assenza di interruzioni della continuità orizzontale e del piano verticale che si sublima nelle falconature; i contrafforti privi di rastremazione nella parte superiore e che rilevano nella composizione architettonica più per gli aspetti decorativi che non come esibizione di un sistema costruttivo.

Un altro scritto ${ }^{18}$ affronta il tema del proporzionamento geometrico dell'edificio. Beltrami dichiara di essersi reso conto della sua rilevanza, un tempo sottovalutata, studiando concretamente il Duomo. L'analisi lo conduce ad affermare, nel confronto tra schemi geometrici diversi, reali o astratti, documentati graficamente, che l'edificio, fin dall'origine, fonda le sue proporzioni sul triangolo egizio e non sul triangolo equilatero che informa il gotico. ${ }^{19} \mathrm{La}$ permanenza dello schema an-

18 La teoria, vedi nota 14 . Anche in questo caso il testo.

19 Luca Beltrami, con una verifica molto analitica riferita alla pianta, agli alzati, al tiburio, dimostra l'effettiva prosecuzione della fabbrica con lo schema geometrico del triangolo egizio che si conferma anche durante le varie fasi di consulenza, comprese quelle di grandi architetti del Rinascimento (Luca Fancelli, Leonardo da Vinci, Bramante, Amadeo, Dolcebuono). Gli architetti del secolo XIV e XV, conclude, sono ben convinti che l'arte si fonda su di una legge interna che non può essere disattesa. Egli rileva anche le differenze nella collocazione della base nell'impostazione geometrica delle chiese tedesche (tangenza alle strutture verticali) e di quelle italiane (sull'interasse delle strutture verticali). 
che durante la fase rinascimentale della costruzione dimostrerebbe che la proporzione nasce da esigenze statiche, ma anche che la pianta e gli alzati che su di essa si elevano, corrispondono ad una intenzione iniziale unitaria. ${ }^{20}$ Quindi il Duomo non è frutto di "sconfinata libertà" come potrebbe apparire per chi ne osservasse la varietà di forme e lo vedesse come un complesso pittorico e disordinato di linee. ${ }^{21}$

E' sulla base di queste considerazioni, della continuità con scelte storicamente accertate, dell'assoluta rispondenza con l'esistente sul piano costruttivo, garanzia di fattibilità e di economia, che Beltrami giustifica il proprio progetto nella relazione che lo accompagna al giudizio della commissione, nelle accorate perorazioni successive alla sconfitta, soprattutto quando la morte di Brentano renderà evidente l'ineseguibilità del progetto vincitore. In uno scritto del $1888^{22}$ egli esamina puntualmente ogni parte costitutiva della fronte per darne giustificazione in rapporto ai principi. ${ }^{23}$

Lo schema geometrico è dunque molto diverso da quello gotico, basato invece sul triangolo equilatero. L'individuazione di una legge formativa intrinseca che permane oltre ogni accidentalità richiama l'idea di Viollet-Le-Duc che fa coincidere il valore d'arte con la totale coerenza delle forme, costruttive e decorative, e d'altronde il testo del secondo scritto si apre con la citazione di un suo pensiero, dal Dictionnaire raisonné d'architecture française du 11 ${ }^{\text {eme }}$ au 16 $6^{\text {eme }}$ siécle (prima Bance, poi Morel, Paris, 1854-1868): "Ceux qui croyent que l'architecture gothique est une exception, une bizarrerie de l'espit humain, n'en ont certes pas étudié le principe, qui n'est autre que la application rigoureusement suivie du système inauguré par les construtions romanes". Beltrami precisa che desume dal volume $4^{\circ}[18 .$.$] , p.21 e possiamo aggiungere: dalla voce Construction. Anche a La teo-$ ria è premessa una citazione, evidentemente funzionale alle sue tesi: “... a tenore della pratica, dell'autorità e della ragione, sembra che le facciate delle Chiese debbeno essere più eleganti e più ricche del rimanente del loro corpo: ma né questo né la simmetria potranno mai permettere che la ragione geometrica del corpo di esse, nella facciata si muti ...". Il corsivo è di Beltrami che aggiunge trattarsi di un "parere inedito di Francesco Croce, a proposito della facciata del Duomo di Milano - Anno 1745".

20 Luca Beltrami svaluta drasticamente la rilevanza della presenza di architetti stranieri, come farà anche in altro scritto, forzando anche l'interpretazione di alcuni documenti, vedi nota 25 .

21 Probabilmente una polemica con Boito, vedremo più oltre le sue tesi, in cui una legittima possibile interpretazione, una lettura del Duomo nei suoi valori pittorici è avvicinata al "disordine di linee" che vi riverbera un senso negativo.

22 Elementi architettonici e decorativi, componenti la facciata del Duomo di Milano secondo il progetto dell'arch. Luca Beltrami, Colombo e Cordani, Milano 1888.

23 I temi trattati sono: la fronte (l'allineamento; la ricerca di verticalità, la strombatura delle porte per permettere l'inserimento delle statue; le corrispondenze con il 
Fra queste valutazioni una appare sconcertante; egli giustifica l'ispessimento centrale della fronte in molti modi fra i quali la possibilità di dare alle porte una strombatura in grado di ricevere statue, come usa nel gotico. Strana affermazione, escluso che si possa pensare ad una inavvertita contraddizione, il che non sarebbe minimamente compatibile con il suo carattere. Dunque il Duomo lombardesco o gotico? La risposta può essere data soltanto se si ricorda il fine di queste analisi, cioè la definizione del progetto della facciata, che si impone sia organicamente conforme alla struttura così come si è storicamente determinata, un problema dunque solo in via secondaria di carattere formale. Il Duomo è struttura lombardesca decorato alla gotica, e questa caratteristica, la sua specificità, deve essere mantenuta. La storia, processo razionale individuante e caratterizzante, non può essere impunemente contraddetta. $^{24}$

capocroce con la realizzazione di una doppia serie di guglie binate; forma dei contrafforti disegnati sul modello di quelli del capocroce ma di maggior dimensione per ottenere l'allineamento con quelli dei fianchi); i contrafforti; il basamento (forma, modalità del risvolto dai lati; interruzioni determinate dalle porte); la questione delle porte (numero, forma arcuata o architravata, con o senza pilastro mediano); finestre; falconatura (rapporto con quella laterale, funzione in rapporto alla identificazione delle navate, rapporti con gli archi laterali di controspinta); guglie; accessibilità della fronte; massima conservazione per la massima economia. Quest'ultimo aspetto non è secondario: l'eccesso di costo è tra i motivi della mancata realizzazione.

24 Una sintesi del senso del progetto di Beltrami è data in forma molto efficace da G. Carotti. Vicende del Duomo di Milano e della sua facciata, in Archivio storico dell'Arte, anno II, 1889, fascicoli III-IV e VII, anche in estratto, Società Tipografico-Editrice Laziale, Roma 1889, p.25., quando afferma che le differenze tra il progetto di Ferrario e quello di Beltrami nel concorso accademico del 1883 segnano lo spartiacque di tutto il dibattito sulla fronte del Duomo. Egli scrive: “... tra i vari progetti presentati segnarono le due correnti dei concetti per la facciata del Duomo. [...] Il bellissimo disegno premiato del prof. Ferrario era un inno spontaneo al maestoso duomo quale era stato sino allora ed ancora interpretato dai romantici dell'arte. Il disegno di grandiosa semplicità e sobrietà dell'arch. Beltrami ... erano la parola dell'arte realistica e del pensiero sperimentale dell'archeologia del secolo nostro. Lo studio entusiastico sì, ma imparzialmente scrutatore e verista delle origini del monumento, delle fasi della sua costruzione, della sue attuali forme e decorazione, era stata la base sulla quale egli aveva incominciato a progettare la facciata del Duomo. Anche Vittore Grubicy, in un articolo sul quotidiano Riforma coglie sinteticamente l'intenzione del progetto di Beltrami scrivendo che egli aveva risolto l'arduo problema della fronte "... esteticamente, scientificamente sotto tutti gli aspetti”, citato da C. Nava. La facciata del nostro Duomo. Dispareri e proposte, Tipografia Pulzato e Giani, Milano 1901, p.6; lo stesso autore cita uno scritto di 
Se questo è il discorso di Luca Beltrami, cioè del principale protagonista del dibattito ma anche delle vicende concrete del Duomo per essere stato più volte architetto della fabbrica, l'antifona è cantata da Camillo Boito, la personalità che guida i concorsi internazionali per la nuova fronte del Duomo, colui che ne determina i destini. Il principale testo di riferimento è pubblicato subito dopo l'esito del concorso di secondo grado. ${ }^{25}$ Nell'esordio alla ricostruzione storica egli scrive che l'e-

Alberto Sormani, sul giornale Ricreazione: "Non mi dilungherò intorno al progetto Beltrami. Dirò soltanto che esso mi ha convinto, mentre il progetto Brentano mi aveva maggiormente conquiso. Da uomo sanae mentis, preferisco la convinzione. Ma amerei anche la conquista per di più."

25 C. Boito, Il Duomo di Milano e i disegni per la sua facciata, Luigi Marchi, Milano 1889. L'opera, senza perdere i caratteri di scientificità mette bene in evidenza le capacità letterarie di Boito che espone i suoi argomenti con vivacità, in una narrazione piena di brio, di ironie che nascondono giudizi anche durissimi, specialmente verso Luca Beltrami. La narrazione storica è accompagnata dalla disanima dei più rilevanti testi a lui contemporanei, con note di dissenso o di consenso. Beltrami, nella esasperata valorizzazione di ogni indizio che potesse attribuire ad italiani i momenti più rilevanti delle fasi costruttive del Duomo, esalta la presenza di Andrea degli Organi e, ritrovata negli annali della fabbrica la nota di una spesa per procurargli due libbre di "morsecate", suppone che si tratti di una corruzione dal francese massicot, e che si tratti quindi di un ossido di piombo, utilizzato per indurire la cera plastica con la quale si fanno modelli. Egli quindi compie una spericolata acrobazia storiografica supponendo senz'altro che Andrea degli Organi avesse dato un modello del Duomo (L. Beltrami. Il Duomo e le sue vicende, in L'Illustrazione Italiana, anno XIV, n.o 39, Milano 11 settembre 1887, pp. 182-191). Boito, citata questa supposizione "del professor Beltrami" elenca in un'intera pagina altri usi dell'ossido di piombo, ed altre interpretazioni del termine, per concludere comunque che ".. del resto il professor Beltrami non è uomo da attribuire troppa importanza alle due libbre di massicot o morsecate, e tira via, fermandosi a più gravi avvertenze". Ancora più severo Boito quando contesta a Beltrami l'attribuzione osservando che non si comprende perché se Andrea degli Organi fosse stato autore del Duomo non ci sia traccia di una sua permanenza nel cantiere e neppure si conoscano altre opere (p. 81) ed ancor più quando rileva, sarcasticamente, un'altra spericolata tesi di Beltrami, che suggerisce la possibilità che l'artista fosse null'altro che Andrea Orcagna. Egli scrive: "Se ne conoscerebbero, in verità, parecchie e stupende [opere di Andrea degli Organi] se fosse vera la dolce supposizione d'un abile architetto, che sospira da mattina a sera attorno al suo Duomo, e ne ha scritto qualcosa e ne ha parlato due volte pubblicamente nel Circolo Filologico di Milano, concludendo il suo secondo discorso con questo scoppio di fantasia: che Andrea degli Organi da Modena fosse, si signori, Andrea di Cione da Firenze detto l'Orcagna, né più, né meno.." (pp. 81-82). Boito rileva che una certa assonanza nel nome non giustifica l'identificazione e soprattutto che dell'Orcagna nulla si conosce dopo il 1368, che è documentata la sua morte nel 1376, mentre il lavoro di 
dificio è di straordinaria bellezza ma che è del tutto incerta la sua nascita per epoca ed autore, che si disputa sul fatto se esso sia italiano, francese o tedesco, ma anche che in un momento storico caratterizzato da una "frantumazione" delle capacità progettuali, dallo "sciogliersi di vincoli", vale a dire dal venir meno del valore esemplare di modelli certi, lo spirito italiano accetta concetti e modi che vengono d'oltralpe, ma li varia, li rende più briosi, meno strettamente logici, meno rigorosamente scientifici. ${ }^{26}$ L'assimilazione di elementi eterogenei gli sembra tanto naturalmente assorbita che forse neppure l'autore ne era consapevole: ciò che appare nella storia del Duomo cioè contrasto e discordia, nella realtà fattuale dell'edificio diventano armonia ed unità. Le osservazioni sull'intervento degli architetti che via via compaiono nei lavori della fabbrica non è mai amplificata oltre il senso stretto dei documenti, tendono a mettere in luce incertezze e contraddizioni, la variabilità delle interpretazioni, l'ambiguità dei termini, ${ }^{27}$ l'irrilevanza dal punto di vista della scienza moderna del dimensionamento geometrico, che riconduce all'esigenza di ottenere una verità attraverso l'apparenza matematica che sostituisca le angosce dell'arte. Molto interessante l'esposizione del dibattito tra il francese Mignot e gli architetti italiani: il primo ritiene corretta l'esecuzione dei piloni di contrafforte a riseghe di cui gli italiani negano l'utilità e afferma l'importanza di una base scientifica alla progettazione, Ars sine scientia nibil est, a cui si contrappone l'affermazione Scientia est unum et ars est aliud. Una contrapposizione che Boito riassume affermando che se Mignot aveva ragione dal punto di vista statico, gli italiani l'avevano dal punto di vista dell'arte, ed a questi vanno le simpatie di Boito. Il modo con il quale egli affronta il tema dell'italianità ci indica con chiarezza una posizione teorica: egli semplicemente osserva che sono evidentemente italiani il Duomo di

Andrea degli Organi per il Duomo data al 1387. Boito prende esplicitamente le distanze dalla seriosità, dalla esibizione dell'erudizione, dal vezzo di dare senso generale ad osservazioni particolari amplificandone il senso; tutto ciò si esprime anche con un'iniziale dichiarazione: i riferimenti bibliografici e documentari saranno dati nel discorso perché l'autore "odia le note" (nell'introduzione, p. XII).

26 La consultazione dell'opera è avvenuta su di un esemplare che era stato di proprietà di Luca Beltrami che evidenzia a margine questi due passi, così come quello citato a nota 25.

27 Per esempio annotando che al termine "triangolo egizio" corrispondono in letteratura figure geometriche di diverse proporzioni, talora anche nello stesso autore (come, per esempio in Viollet-Le-Duc). 
Orvieto, di Siena, così come sono evidentemente di gotico francese quelli di Strasburgo, o di Parigi; il Duomo di Milano non ha patria, nasce nei contrasti, ha una propria forza, una propria originalità. Boito non accetta ipotesi non confortate da riferimenti oggettivi: non ci sono disegni della fronte o prove documentarie della sua esistenza; l'ipotesi corretta è che non sia mai esistito un disegno; non ritiene, come altri, che il disegno del Duomo inserito nella sua opera da Cesariano ${ }^{28}$ sia derivato da un documento più antico; si esprime contro i "sottili ragionatori" ed avverte che "i ragionamenti a fil di spada fanno dire le grandi corbellerie". ${ }^{29} \mathrm{Ma}$ soprattutto egli si esercita nelle valutazioni critiche delle antiche proposte per una fronte, considerando del tutto negativo quanto prodotto sia in epoca barocca sia in epoca neoclassica ${ }^{30}$ mentre sono apprezzati gli interventi cinque e seicenteschi, ed ancor più taluni elementi più antichi, come le finestre, ${ }^{31}$ il piede dei contrafforti. E' all'interno di queste considerazioni che Boito introduce un argomento nuovo che avrà in seguito grande rilevanza: egli considera la qualità delle "impronte" lasciate dal ' 500 , dal '600, dall' 800 , osserva che un secolo è sufficiente per attribuire un'importanza storica, e che quegli elementi hanno oramai due o tre secoli di vita, che il momento attuale ha scrupoli di conoscenza, anche per le cose più secondarie. Egli sembra ricordare se stesso, le proprie tesi sul restauro, ${ }^{32}$ prendere atto del valore del monumento come documento di una pluralità di epoche, soprattutto del valore di antichità, non senza mostrare le sue preferenze

28 V.L. Pollione, (C. Cesariano), Di Lucio Vitruvio Pollione de architettura libri dece traducti de latino in vulgare raffigurati: commentati..., Da Ponte, Como 1521, libro I, foglio XIV.

29 C. Boito, Il Duomo di Milano e $i$ disegni per la sua facciata, Luigi Marchi, Milano 1889 , p. 265.

30 La proposta più violentemente criticata è quella del Castelli, un ibrido senza senso, con una censura che colpisce anche il Bernini che lo aveva approvato, ma severamente criticati sono tutti i progetti settecenteschi specialmente quelli di Merlo, Galliori, Vittone, Juvara, il pensiero dello "scipito e sgangherato Vanvitelli" (p. 265).

31 Eccettuata quella centrale, del Soave, "soavemente pedantesca" (p. 268).

32 Egli era stato propositore nel convegno nazionale degli architetti ed ingegneri italiani del 1883 di un documento, unanimemente approvato, nel quale si enunciavano i principi di un restauro che riconoscesse il valore diacronico della testimonianza costituita dal monumento, e quindi la necessità di rinunciare all'unità stilistica per rispettare ogni stratificazione di rilievo storico o artistico, vedi Atti del quarto congresso degli ingegneri ed architetti italiani, tipografia Fratelli Centenari, Roma 1884

33 Nella "vecchiaia rispettabile" si apprezza il barocco ed il rococò e "persino il 
di stile. ${ }^{33}$ Tuttavia non ritiene sbagliato dare al Duomo una nuova fronte: quella enorme, meravigliosa sintesi di interventi di epoche diverse, rappresenta un concetto di grande unità, salvo che nella fronte. ${ }^{34}$ Infine, argomento decisivo, i milanesi la vogliono. ${ }^{35}$

I principi ai quali il progetto deve ispirarsi appaiono nelle valutazioni degli elaborati dei due concorsi internazionali, che egli inserisce nella narrazione degli eventi, unendo fra loro i larghi principi ispiratori del bando di concorso ${ }^{36}$ (che lasciava massima libertà espressiva, imponendo soltanto l'uso del Candoglia e l'organico collegamento con il corpo dell'edificio $)^{37}$ ai concetti espressi nelle relazioni, alle considerazioni della giuria, alle proprie (Fig. 4, Fig. 5, Fig. 6, Fig. 7).

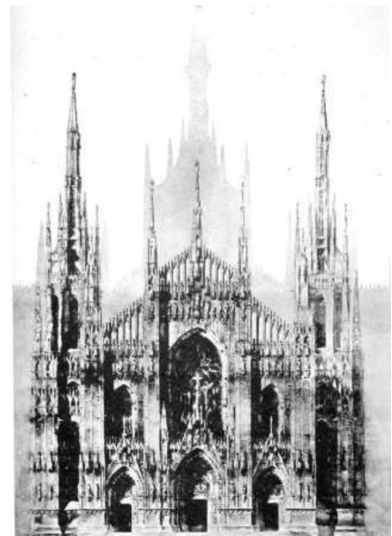

Fig. 4. Il progetto di Giuseppe Brentano, concorso internazionale di primo grado per la fronte del Duomo dal volume di Camillo Boito, Il Duomo di Milano, Milano 1889, tavola 51.

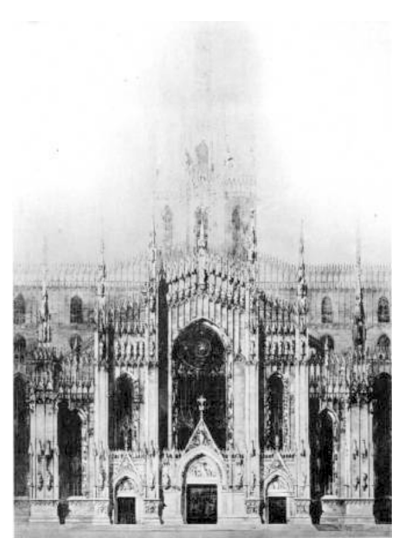

Fig. 5. Il progetto di Luca Beltrami, concorso internazionale di primo grado per la fronte del Duomo dal volume di Camillo Boito, Il Duomo di Milano, Milano 1889, tavola 47.

primo impero" e certamente quello stile è richiamato per il fatto che al momento il secolo non si era ancora compiuto, ma è presente anche un sottinteso negativo. (p. 268-269).

34 Boito umanizza l'edificio: corpo che "sanguina nel viso", cui "manca una faccia”. (p. 269).

35 Si citano qui in particolare le donazioni Mellerio e De Togni. (p.269).

36 Il bando, emesso dalla fabbriceria, è sostanzialmente ispirato da Boito, ma sembra che Luca Beltrami, che era architetto della fabbrica, prima di dimettersi per partecipare al concorso, vi abbia apportato non indifferenti variazioni.

37 L'assenza di un limite di costo, giustificata nel bando con la volontà di non limitare le capacità espressive dei concorrenti, deriva anche da una fiducia nella possibilità di raccoglier gli ingenti capitali occorrenti. 


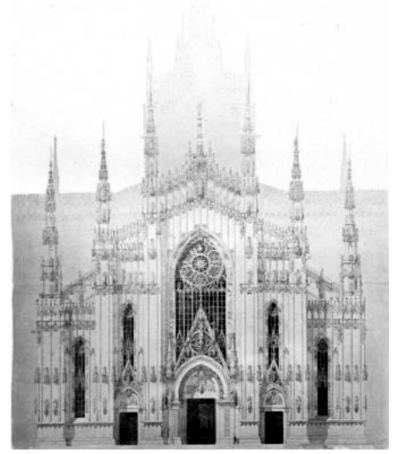

Fig. 6. Il progetto di Giuseppe Brentano, concorso internazionale di secondo grado per la fronte del Duomo dal volume di

Camillo Boito, Il Duomo di Milano, Milano 1889, tavola 83.

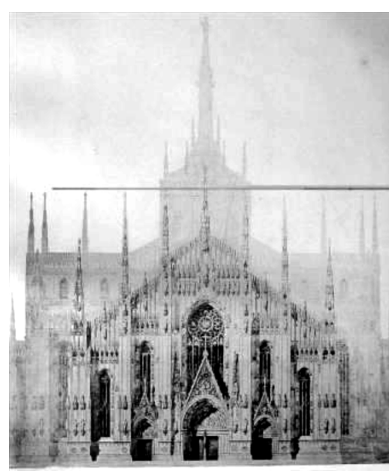

Fig. 7. Il progetto di Luca Beltrami, concorso internazionale di seondo grado per la fronte del Duomo dal volume di Camillo Boito, Il Duomo di Milano, Milano 1889, tavola 81.

In sintesi le conclusioni sono: che il Duomo è monumento unico; che la varietà e la complessità delle sue forme costituiscono uno stile; che è forma di arte "paesana" ma sotto la viva influenza straniera; che l'unicità impedisce che ci si possa ispirare, se non con grandissima cautela, ad edifici anteriori o coevi; che l'assenza di principi di indirizzo nel bando determinava la necessità di un giudizio non sui criteri adottati ma sulla qualità della loro applicazione da un punto di vista artistico. ${ }^{38}$ Un'impostazione puramente estetica, che sembra condivisa dalla maggioranza della commissione ( $\mathrm{ma}$ i verbali sono troppo sintetici per una sicura individuazione $)^{39}$ ribadita fortemente nel bando di secondo grado in cui si esclude la possibilità di dare indicazioni sui campanili (sarebbe come fare l'opera) anche perché la storia, che è imparziale, non ha ancora dato un verdetto sicuro nel definire il Duomo italiano o tedesco, non si vogliono dare limitazioni agli invi-

38 Boito esemplifica gli esiti del primo concorso: fronti con e senza campanili; con campanili sulle navate minori o le centrali; a due pendenze o a pendenze spezzate sulle navi; pendenze parallele o convergenti; con cuspide o orizzontalità del finimento; con torri snelle o schiacciate; con un finestrone centrale o un rosone; fronte rettilinea o con avanzamento della parte centrale realizzato con la massa muraria o con il portico. (pp. 271 e seguenti, in particolare 272).

39 A pp. 271-274. 
tati che sono "maestri" ${ }^{40}$ Boito sintetizza quei principi e li fa propri: predominio dell'arte sulla critica, base di studi storici purché non giungano a conclusioni dogmatiche; cooperazione di arti diverse, italiane ed oltramontane; aderenza alla speciale fisionomia del monumento.

Trascurando l'esame dei giudizi, che assegnano la vittoria a Brentano con un apprezzamento particolare per Beltrami, Boito conclude la sua monumentale opera con un capitolo dedicato allo stile. Lo scritto si apre con un sintetico slogan che l'autore dichiara essere la sintesi del suo pensiero: "L'occhio vuole la sua parte" ${ }^{41} \mathrm{ma}$, avverte, l'occhio è educato e sintetizza istantaneamente l'insieme delle conoscenze attuali e storiche. Il sentimento può errare ma il ragionamento non è infallibile, "soprattutto quando nelle questioni del bello usa alambicco e storta". ${ }^{2}$ Lo stile di un paese, di un monumento, si può studiare analiticamente (provenienze, reciproche influenze e così via) ma sussiste infine un certo non si sa che, che egli chiama espressione, che si può capire solo se si ha il cervello sgombro da teorie assolute ed esclusive. ${ }^{43}$ La sua conclusione rispetto alle vicende del Duomo è che esso nasca da un disegno primitivo di origine straniera, che gli italiani l'abbiano modificato ancor prima di incominciare, che nel momento in cui si trovavano impacciati si rivolgevano agli stranieri, che alle prime alterazioni più che all'esito dei dibattiti e dei contrasti si deve la bellezza dell'edificio. L'analisi esasperata per mostrarne le differenze dal gotico è inutile perché già fatta da Förster. ${ }^{44}$ Sono esplicitamente citati Luca Beltrami, Giulio Carotti, e soprattutto Aristide Nardini Despotti Mospignotti di cui cita

40 Le restrizioni riguardano la richiesta di una fronte intimamente connessa con l'organismo; il divieto di presentare disegni pittorici, ammesse solo le ombre destinate ad evidenziare i volumi, disegni a solo contorno della fronte (1/50), della pianta e delle sezioni $(1 / 100)$, una prospettiva a scala libera.

41 A pp. 279.

42 Ivi.

43 Boito non manca di fornire esempi storici (pp. 280-281): dalle contraddizioni di Francesco Milizia che sprezza il Duomo (in Memorie degli architetti antichi e moderni, Cardinali e Frulli, Bologna 1827) eppure loda il gotico (in Principj di architettura civile, Remondini, Bassano 1813) o il Barca, che loda il Duomo, ma si rammarica che non sia di architettura vitruviana, e rivendica alla modernità (Boito cita uno scritto del 1607, non meglio precisato e non rintracciato).

44 A p. 283 Boito cita da Förster, 1866, senza indicare titolo, che scrive "...malgré tout ce qui a été italianisé, il subsiste assez de l'ésprit et des idées de l'architecture 
la tesi che vorrebbe in San Pietro in Ciel d'Oro ed in Sant'Ambrogio i precedenti del Duomo. ${ }^{45}$ Boito si oppone alle interpretazioni "razionali" citando un razionalista: Viollet-Le -Duc che afferma essere lo stile "manifestation d'un idéal établi sur un principe"; ${ }^{46}$ chi potrebbe confondere l'ideale del lombardesco con quello del Duomo. Esso è caratterizzato da contrafforti, pinnacoli, archi rampanti, finestroni altissimi e nervati, piloni che si ramificano, dal giro delle arcate nel presbiterio. Cosa importa se mancano le cappelle intorno all'abside, che i capitelli siano a tabernacolo, che le finestre lascino un poco di parete? Sono dei particolari ".. se lo stile è un ideale e se il sentimento mi alza a questo ideale?" ${ }^{47}$

Gli strali a Beltrami sono molti, di massima evidenza, anche quando non lo si nomina; Boito afferma che è "curiosa evoluzione quella che riguarda un individuo solo", ma anche che se si trattasse del "cosiddetto principio degli adattamenti", ${ }^{48}$ non si comprende perché la morte di qualche maestro avesse potuto provocare una profonda dimenticanza dei principi, della stessa legge, e si procedesse rompendo ogni legame di scala e di proporzione, con il continuo bisogno di cercare all'estero un conforto magistrale. Boito mette

allemande pour qu'on puisse, des qu'on passe a l'èxamen, remarquer tout ce qui s'en carte." la citazione proviene da E. Forster. Monuments d'architecture, de sculture et de peinture de l'Allemagne depuis l'établissement du christianisme. Tome troisiemme, Morel, Paris 1866.

45 Le tesi espresse da A. Nardini Despotti Mospignotti. Del Duomo di Milano e della sua nuova facciata - Studi, in "Il Politecnico - Giornale dell'Ingegnere Architetto civile ed industriale, vol. XXXVI, 1888, pp. 685-709, Ivi, vol. XXXVII, 1889, pp. 286315 , ma anche in estratto, Saldini, Milano 1889, sono a favore dell'italianità del Duomo, fin dalle sue origini, benché ciò non sia comprovabile; la sua tesi è che il primo progetto, sia di origine francese, voluto e procurato da Gian Galeazzo Visconti, ma subito respinto dai milanesi; egli lo colloca molto prima della data di presunta fondazione, il 1386, che ritiene invece momento di una radicale trasformazione di un precedente progetto. Boito rileva una sostanziale contraddizione nelle sue tesi, poiché se nell'articolo citato si esprime a favore dell'italianità del Duomo, con espressioni molto nette, in un successivo articolo, come artista, reclama una fronte: che non segua i difficili ragionamenti degli eruditi; soddisfi "il volgo", che lo vede gotico; che arieggi quindi il nordico.

46 A p. 285; Boito non indica il volume da cui trae la citazione, ma si tratta di: E. Viollet-Le-Duc. Dictionnaire raisonné d'architecture française du 11. au 16. siécle, prima Bance poi Morel, Paris, 1854-1868, alla voce Style, vol. ottavo (1866) p. 479.

47 p. 285.

48 p. 295. 
in luce che nella stessa epoca altri edifici crescono bellissimi senza conforto di maestri stranieri, primo fra tutti la Certosa di Pavia ${ }^{49}$ Non mancano i riferimenti di natura storica e documentaria che dimostrano la volontà di ottenere pareri per l'ignoranza italiana sul tipo di edificio che si andava costruendo..$^{50}$

Ma soprattutto egli rifiuta le tesi dei "critici" che vorrebbero spiegare le forme del Duomo come conseguenza dei materiali (citando chiese simili nello stile ma fatte di materie diverse come Limoges, ClermontFerrand, Narbonne) o dell'ampiezza (dimostrando che era possibile ottenerla anche con un vero lombardesco) o come esito di una intenzione di novità, in questo caso contestando la tesi a Giuseppe Brentano ${ }^{51}$ che accusa di voler interpretare il passato, che non aveva smania di nuovo, con i sentimenti di oggi; lo stesso argomento è contestato a Cantừ ${ }^{2}$ che attribuisce il desiderio del nuovo all'azione dei maestri campionesi. Boito nega che le consorterie portino novità, piuttosto consolidano lo stile; nega che esistessero effettive compagnie organizzate, specialmente campionesi, esemplificando con le vicende del Duomo di Monza.

49 Boito, che non rinuncia mai ad una scrittura narrativa discorsiva e ricca di annotazioni estemporanee, scrive a proposito delle prime fasi della costruzione (p. 296): "Codesto parto, che alcuni critici d'oggi proclamano di sangue tutto lombardo, appena svezzato non sapeva muovere le gambe, mettere la pappa in bocca, adattarsi sul capo il cercine senza sentirsi accanto il balio francese o tedesco; e diventato grandicello, poi giovinotto vigoroso e formosissimo, continuò a provare tuttavia la necessità di pedagoghi e precettori stranieri, mentre la Certosa di Pavia e tutti gli altri belli edifici contemporanei nascono e crescono e mettono i baffi e poi generano senza ricorrere a nessuno di là dei monti e dei mari."

50 Non manca un conforto documentario nel discorso tenuto da Roberto Barbavara, funzionario ducale, ai fabbricieri, in cui si attribuiscono i difetti della fabbrica all'imperizia dei tecnici preposti a fronte di un edificio che costituiva una novità rispetto alle tradizionali costruzioni lombarde.

51 Si veda: G. Brentano, Concorso internazionale di II grado. Per la nuova facciata del Duomo di Milano. Archietto Giuseppe Brentano MDCCCLXXXVIII, Ricordi, Milano 1888.

52 Si veda: C. Cantù, Gli annali della fabbrica del Duomo di Milano, in Memorie del Reale Istituto Lombardo di scienze e lettere, vol. XVI, pp. 203-213, Milano 1885 , ma in precedenza, in forma ridotta con il titolo Le origini del Duomo nel periodico La Sapienza, vol. XII, Torino 1865 e su La Perseveranza, Milano 24 novembre 1885; il testo non è altro che il Proemio che Cantù aveva redatto, assieme a note illustrative, per gli Annali della fabbrica del Duomo di Milano dalle origini fino al presente, pubblicati per cura della sua amministrazione, Brigola, poi Tipografia sociale, poi Reggiani, Milano 1877-1885. 
Infine, in questo caso in polemica con tutti gli autori precedentemente citati, Boito afferma che occorre dar credito anche alle tradizioni orali, come quella trasmessa dal Ceruti ${ }^{53}$ che cita una relazione del XVI secolo che riferisce di una delegazione di 24 notabili milanesi che si recano all'estero alla ricerca di un disegno di grande magnificenza; lo stesso autore ${ }^{54}$ afferma l'esistenza di un disegno di origine tedesca $^{55}$ messo subito in modello, ed infine il Cesariano ${ }^{56}$ che tratta di un disegno tedesco tradotto in modello in seguito andato bruciato, e su questa tesi, egli ricorda, sono concordi anche il Mongeri, Schimdt e De Dartein, di cui accetta l'opinione che il progetto originario abbia subito profonde modificazioni. ${ }^{57}$

L'esame del dibattito fra gli storici e gli architetti che partecipano al concorso mostra una infinità di varianti, sia nella individuazione dell'area culturale che costituisce la matrice originaria, sia della propensione ad un progetto basato su valutazioni di congruenza organica o di invenzione formale. ${ }^{58}$ Una affermazione molto recisa dell'italianità del Duomo, con qualche definizione generale poco condi-

53 A. Ceruti, I principi del Duomo di Milano sino alla morte del Duca Gian Galeazzo Visconti, Agnelli, Milano 1879.

54 Idem, citando una Descrizione storica delle chiese e dei monasteri milanesi, vedi nel volume di Boito a p. 304.

55 Boito mette chiaramente in luce i rapporti politici di Gian Galeazzo Visconti con il mondo tedesco e con la Francia.

56 Vedi nota 28.

57 F. De Dartein, Etude sur l'architecture lombarde et sur l'origines de l'architecture romano-byzantine, Dunod, Parigi 1865-1882; F. Schmidt. Il Duomo di Milano... conferenza tenuta all'adunanza ... degl' Ingegneri ed architetti austriaci in Vienna, il 10 aprile 1886 ..., in Il Politecnico, anno XXXIV, Milano 1886, pp.429-437; G. Mongeri. Per la facciata del Duomo di Milano,1887. Memorie e commenti, Tipografia degli ingegneri, Milano 1887.

58 P. Cesa Bianchi, Alcune considerazioni unite ai progetti presentati al concorso di secondo grado per la Nuova Facciata del Duomo di Milano, Pirola, Milano 1888, che sostiene la derivazione estera del Duomo, con riferimento soprattutto all'architettura renana, del Finisterre, del Nord della Francia, delle Fiandre, della stessa Inghilterra del sud, anche per influenza dei lapicidi lombardi che in quei paesi avevano lavorato. Egli, tecnico della fabbriceria, sostiene l'ottima qualità costruttiva dell'edificio, nei pilastri, nelle murature e soprattutto della fronte. Le proposte muovono da ipotesi di continuità: l'abside gli appare di bellezza assoluta e quindi modello per la fronte, in particolare per le finestre; il basamento elemento architettonico da risvoltare; le cinque navate una particolarità da esaltare con cinque porte nella fronte; le porte da farsi gemine sul modello di quelle d'oltralpe che ha tuttavia esempi in Italia (Sicilia, Campania, Emilia). 
visibile, si trova nello scritto di Antonio Caregaro Negrin..$^{59}$ Egli fa riferimento allo stile archiacuto, uno stile religioso "detto impropriamente gotico" le cui caratteristiche sono date da un tentativo di smaterializzazione dell'architettura, un voler far svanire la materia, cui corrisponde lo sforzo morale dell'elevazione. Il Duomo ne sarebbe una versione innestata su di un organismo italiano la cui progettazione attribuisce ai maestri comacini e campionesi con influenze dell'area nord-occidentale dell'Europa; egli scrive sulla scorta principalmente delle affermazioni di Federico Schimdt ${ }^{60}$ e di Förster ${ }^{61}$ ma citando anche, per gli aspetti generali della storia dell'architettura, Selvatico e Mongeri. Le caratteristiche italiane sono individuate nel sistema costruttivo dichiarato uguale a quello delle altre chiese "Italiane Lombarde del medesimo tempo; nel fatto che i maestri campionesi e comacini hanno sì utilizzato l'arco acuto, ma con modalità loro proprie, senza perdere un legame con l'antichità classica; nella forma della copertura; nella disposizione dei peducci rispetto alle arcature della finestra; nella sporgenza dei pilastri. Egli ritiene in definitiva che le presenze straniere siano state del tutto effimere e che la chiamata del Gamodia, di Mignot e di altri fosse da considerare un vero e proprio errore, e, concordando in questo con lo Schmidt ${ }^{62}$ e con Mongeri ${ }^{63}$ afferma che fino alle volte la costruzione sia stata del tutto italiana. La forma della nuova facciata dovrà ispirarsi alle stesse forme

Egli comunque presenta un progetto senza torri ed uno che le prevede, proponendo anche l'ipotesi della loro realizzazione in un secondo tempo; A. Guidini. La facciata del Duomo attraverso $i$ secoli e gli attuali concorsi, in Illustrazione Italiana, Treves, Milano 11 settembre 1887, numero speciale intitolato Il concorso mondiale per la Nuova Facciata del Duomo di Milano; l'autore compie un'accurata disanima dei pregi e degli inconvenienti tecnici delle due soluzioni, ma sembra infine propendere per una fronte senza campanili.

59 A. Caregaro Negrin, Il duomo di Milano non è monumento tedesco o francese ma italiano. A proposito del grande concorso internazionale per una nuova facciata, Tipografia Paroni, Vicenza 1887 (lettura fatta all'Accademia Olimpica in adunanza pubblica ...28 gennaio 1887).

60 Vedi testo citato a nota 57; da Schmidt si riprende il concetto della loro operosità nella valle del Reno e si cita l'opinione che si trattasse di vere e proprie società in cui l'individuo, qualunque fosse la sua capacità, era assorbito.

61 Vedi nota 44.

62 Vedi testo citato a nota 57.

63 Vedi testo citato a nota 57 
del Duomo, non escludendo comunque la possibilità di inserire un campanile l'importante è eliminare gli interventi incongrui e ricondurre il Duomo ad unità. ${ }^{64}$

Tra le opere degli storici si distingue per l'accuratezza e la chiarezza delle posizioni Giulio Carotti ${ }^{65}$ che antepone al suo testo un vero e proprio programma di studio al fine di individuare quali siano i caratteri dell'edificio, muovendo dalla conoscenza dell'architettura lombarda.$^{66}$ Le considerazioni, che lo portano a definire la sostanziale italianità dell'edificio sono molto circostanziate soprattutto nei puntuali confronti tra edifici, e seppure poco differiscano da altre già viste, sono più precisamente documentate.$^{67}$ Egli è anche tra $\mathrm{i}$ pochi che escono dal dibattito strettamente riferito al Duomo facendo riferimento a Burckhardt che aveva esaminato l'attività degli architetti te-

64 Mirare "... quindi a togliere lo spettacolo delle incondite impronte lasciate dai tempi infausti che il paese crescente a coltura non potrebbe più a lungo tollerare." (p.20).

65 G. Carotti, Il Duomo di Milano e la sua facciata, Tipografia Bortolotti di Giuseppe Prato, Milano 1888.

66 Il testo di riferimento è quello di Ferdinand De Dartein, vedi nota 57.

67 In particolare sono ritenute prove di italianità: la planimetria che confronta con quelle di Amiens e di Colonia, erroneamente giudicati precedenti; afferma rilevanti le somiglianze strutturali con Sant'Ambrogio, che ritiene di cinque secoli più antico, a sua volta ritenuto più simile al Duomo di quanto non lo sia con le basiliche romane o ravennati; la semplicità delle linee perimetrali; la sporgenza limitata dei contrafforti, la loro configurazione senza riseghe; l'atrofia degli archi rampanti esterni; lo sviluppo in lunghezza dell'asse maggiore; la configurazione generale della massa; il transetto poco sporgente, come in San Michele di Pavia; la presenza preminente nell'organismo di un tiburio con uno sviluppo eccezionale, sconosciuto all'estero, sviluppo di Sant'Ambrogio, di San Teodoro, di San Michele e di San Pietro in Ciel d'Oro di Pavia; l'andamento del tetto con una falda per navata; un elevato con proporzioni che non sono gotiche; $i$ piloni, che hanno già forma composita nel romanico e che nel Duomo si presentano con un perimetro continuo, con raccordi curvilinei, un andamento dolce al contrario di quelli gotici, specie a Colonia a cui si fa riferimento come precedente del Duomo, dove essi hanno tagli secchi, taglienti. Ma Carotti si spinge più in là quando afferma che non può essere considerata di derivazione estera la configurazione a cinque navate, nuova in Italia ma presente in Francia soltanto nel Saint Saturnin di Tolosa e comunque pressoché irrilevante, dato che in origine si pensava ad una chiesa a tre navate con grandi cappelle laterali; analoga la sua posizione in rapporto alla forma absidale: la si dice estera ma la si ritrova soltanto a Santa Croce di Gmund, San Lorenzo di Norimberga; anche per le finestre si indica uno sviluppo dal primo apparire di archi acuti alla loro permanenza nell'architettura lombardesca. 
deschi in Italia il cui gotico si trasforma e passa "... dal principio fondamentale del gotico del nord (cioè l'organismo ... tendente a grande elevazione con sviluppo di forze contrastanti) al concetto meridionale di vasti spazi e grandi masse con il predominio della massa orizzontale"; ${ }^{68}$ così il gotico nordico utilizza quasi come un'insegna la torre, che non è invece ritenuta necessaria in Italia. Dunque il carattere straniero del Duomo si limita all'aspetto esteriore. Da qui le indicazioni per la nuova fronte: occorrono architetti che siano artisti, ma che si lascino guidare meno dall'entusiasmo e più dall'osservazione archeologica, senza di tentare di gareggiare con l'innavicinabile bellezza delle parti absidali: il Duomo ha bisogno di una grandiosità che non sia esuberanza decorativa. Si noti: Carotti loda Tito Vespasiano Paravicini riconoscendo che per primo egli aveva rivendicato il carattere lombardesco del Duomo. ${ }^{69}$

I protagonisti dei concorsi, cioè gli architetti, che talora hanno pubblicato le loro relazioni accompagnatorie, si basano in genere su valutazioni puramente formali, rare le giustificazioni di natura teoretica. Fra coloro che si distinguono per approfondimento Giuseppe Brentano, forse anche per la sua fresca laurea al Politecnico, per le relazioni mantenute con i suoi maestri, Boito e Beltrami. ${ }^{70}$ Egli dunque, nel testo che accompagna il progetto di secondo grado ${ }^{71}$ premette un'analisi storica per la quale dichiara di far riferimento ai testi di Nava, Cantù, Beltrami, Cesa Bianchi, Ceruti ed alla "...brillantissima monografia di Boito" ${ }^{72}$ che, assieme alle valutazioni formali, lo conduce a dichiarare che non sussistono documenti che comprovino l'idea di erigere delle torri; che non è possibile, se non con contrafforti inaccettabili, elevare a torri i pilastri angolari quadrati; che esse costituirebbero comunque un'alterazione grave dello schema planimetrico, introducendo una frattura con l'interno dando rilievo a tre sole navate nella fronte e riducendola così allo schema del capocroce, senza la necessaria prevalenza ge-

68 In Carotti, op. cit. a nota 65, che annota provenire la citazione da J. Burckardt. Der Cicerone, 5 a edizione a cura di Guglielmo Bode, Leipzig, 1884, vol. II, p. 44 e seguenti.

69 Si riferisce all'articolo qui citato a nota 4; Carotti segnala anche l'opposizione di Cesa Bianchi, vedi citazione a nota 5 , e di Boito.

70 Non mancherà fra i due qualche polemica nella rivendicazione di quella maestranza; gli archivi ci restituiscono tuttavia alcune fotografie dei suoi disegni della fronte del Duomo con una dedica a Beltrami.

71 Vedi riferimento bibliografico alla nota 51.

72 Vedi alle note 5, 11, 24, 52. 
rarchica. Egli dunque giustifica il progetto di primo grado, che presentava due grandi torri, come una prova, non l'idea primitiva ma un desiderio di studiare il Duomo anche da questo punto di vista. ${ }^{73}$ Una presa di distanza dal primo progetto che si accompagna all'accettazione delle tesi di fondo di Beltrami, da cui si distacca nel sostenere che far sporgere la parte centrale della fronte non avrebbe dato maggiore imponenza, sarebbe stato come ridurre la chiesa ad una forma a tre navate, avrebbe creato una massa centrale con due miseri rinfianchi con un effetto tipico di architetture moderne e non dell'antico.

Una tesi sostanzialmente alternativa a quelle qui presentate proviene da Henry de Geymüller ${ }^{74}$ il cui scritto ha dichiaratamente lo scopo di dare consigli ai milanesi perché compiano una fronte degna della grandezza del monumento ${ }^{75}$ non essendo a suo parere soddisfacente alcun progetto fra i molti fino ad allora presentati. Egli ricorda come le cattedrali di Firenze, Bologna e Milano siano sorte contemporaneamente $^{76}$ e ritiene che ciò abbia dato luogo ad una vera e propria competizione fra le città, per affermare attraverso la Cattedrale una supremazia intellettuale e politica. La certezza, data dagli Annali della continua ricerca da parte di Gian Galeazzo di esperti architetti tedeschi, tenuto conto dei suoi vasti programmi edilizi, della capacità della cultura locale di impostare grandiosi edifici, come la Certosa di Pavia, esclude una ricerca casuale o determinata dalla incapacità degli architetti disponibili in luogo. rivolgersi a stranieri aveva lo stesso significato che ebbe a suo tempo la chiamata di Bernini in Francia da parte di Luigi XIV, per il Louvre, o quella di Pellegrino Tibaldi in Spagna, per l'Escoriale. Egli la

73 Questa versione appare poco credibile anche se Brentano aveva effettivamente presentato al concorso di primo grado due progetti, uno a capanna ed uno a torri laterali, che appare però del tutto definito e ben al di là di una semplice prova, ed in ogni caso è quello che gli merita il passaggio alla prova di secondo grado.

74 H. De Geymüller, Le passè, le present et l'avenir de la Cathédrale de Milan, in Gazette des Beaux Arts, Parigi febbraio 1890, pp. 152-161, marzo 1890, pp. 208-221, aprile 1890, pp. 307-321; l'articolo è pubblicato anche in estratto, senza data.. Egli ribadisce tesi che dice già espresse in precedenza affermando che la pubblicazione degli Annali, pur dando molte notizie nuove (di cui dà resoconto), modificando credenze consolidate sulle origini del Duomo, non ha alterato le sue tesi di fondo.

75 De Geymüller loda l'attività della fabbriceria, la pubblicazione degli Annali, il bando dei concorsi internazionali, ma ritiene che nessun progetto sia soddisfacente, perché non si è tenuto conto del carattere eccezionale del monumento.

76 Rileva anche che, a distanza di cinque secoli, si pone contemporaneamente il problema della costruzione della fronte. 
interpreta come una volontà di differenziazione, anzi di contrapposizione con Firenze: una precisa volontà di ricercare un linguaggio diverso. Accanto ad analisi sulla genesi di San Petronio, sulle possibili analogie tra il Duomo e San Lorenzo, stanno alla base delle sue argomentazioni la documentata intenzione del Seregni nel 1537 che afferma di corrispondere all'intenzione dei primitivi costruttori, la cui intenzione sarebbe dimostrata anche da un disegno di Leonardo, da pitture nell'appartamento di Leone X nel palazzo della Signoria di Firenze; egli si dichiara ancora d'accordo con Mongeri quando afferma che Cesariano presenta qualcosa che non è sua invenzione, ma proviene da documenti che lo precedono, ma anche che egli dipenda dalle cognizioni trasmesse dal suo maestro, Bramante. Con questo stabilisce una continuità nell'ipotesi storica che per la fronte fossero previsti campanili.

Egli suppone una emulazione tra le città italiane che ha una sua cronologia, perché è Firenze che stupisce il mondo con la cupola di Brunelleschi, è San Petronio che si presenta con un organismo più aggiornato e, come dimostra il modello in legno della chiesa, con uno straordinario complesso nei bracci di croce, con quattro campanili alle testate dei transetti a circondare la cupola, quasi una ripresa dello schema di San Lorenzo. Milano non poteva inserirsi in questa gara architettonica se non con una facciata grandiosa.

L'origine tedesca non esclude la profonda trasformazione dello schema, della forma strutturale secondo una visione dell'architettura propriamente italiana ${ }^{77}$ ma quest'ultima non esclude affatto la presenza dei campanili, come documentano le moltissime costruzioni, e gli ancor più frequenti progetti, presenti nella tradizione italiana. ${ }^{78}$ Se il Duomo è infine costruzione originale tuttavia non gli appare difficile ritrovare nei singoli elementi l'influenza tedesca ${ }^{79}$ puntualmente indicate e che

77 Secondo Geymüller quindi, pur non essendo possibile indicare un architetto “originario", forse neppure esistito tenuto conto del fatto che la figura dell'architetto di quel tempo era molto diversa dalla moderna, ritenuta risibile la rilevanza di figure come quella del Gamodia o del Bonaventura, dichiara tedesco senza dubbi il primo impianto del Duomo: per il ravvicinamento dei pilastri, il tracciato dell'abside.

78 Sono citati Filarete, Bramante, i Sangallo, Cristoforo Rocchi.

79 Si tratta di una risposta implicita a Luca Beltrami, con cui aveva ed avrà costantemente in futuro un amichevole rapporto e di cui loda l'idea di costruire un campanile che accolga le porte e le finestre pellegriniane; si noti che il testo di Geymüller si rivela aggiornatissimo nella conoscenza della contemporanea bibliografia italiana. 
gli appare rilevantissima; il continuo richiamo di architetti dall'estero è per lui prova del fatto che gli ingegneri italiani sono consapevoli che il progetto iniziale era basato su di uno stile straniero, e che essi non erano totalmente in grado di dominarne lo sviluppo. ${ }^{80}$ Altrettanto puntualmente Geymüller indica le similitudini con monumenti italiani per concludere con Burckardt ${ }^{81}$ che la cattedrale non è francese, tedesca o italiana, ma un unicum, in cui di latino vi sono la semplicità, la chiarezza, l'ampiezza, la bellezza delle proporzioni molto serene.

Nel giudicare la fronte moderna egli afferma che essa ha due meriti: le porte e le finestre, quindi due interventi di altro stile, ed il fatto che essendo piccola rende affascinante l'impatto con il grandioso interno. Egli si pone il quesito se sia legittimo fare una fronte nuova: la risposta è positiva purché si salvino i bellissimi inserti rinascimentali, la si studi in rapporto formale con la piazza. Le osservazioni sulle fronti sono guidate da questi due obiettivi di fondo ma infine egli sostiene che fino al 1883 non si discute della fronte del Duomo senza pensare alle torri, che solo da quella data si pone l'assimilazione gotico/ torri lombardesco/ assenza di torri, con la conseguente lotta nel primo concorso internazionale. ${ }^{82}$ Il fatto che Milano abbia l'unica Chiesa con quella forma non per questo la fa meno italiana. In sostanza Geymüller supera la contrapposizione, esce da una correlazione diretta tra nazione e stile per riferirsi piuttosto al caso concreto, ad una storia del Duomo che si emancipa dalle sue origini divenendo sempre più italiana.

Infine Geymüller si oppone alla applicazione di un metodo logico rigoroso alle questioni dell'architettura (" ...danno alla logica le chiavi della loro anima..."). Principi razionalisti possono divenire il vanto della modernità (profeticamente!) ma sono inapplicabili all'arte gotica, con un evidente riferimento a Viollet-Le-Duc nella prima esposizione della tesi che diviene poco oltre esplicita. Egli dunque rifiuta l'idea affermata da Beltrami, seguita da molti concorrenti del secondo grado di concorso, e

80 Geymüller fa riferimento a Fergusson senza indicare un testo specifico, forse: J. Fergusson. History of architecture in all countries: from the earliest time to the present day, Murray, London 1873-1876.

81 Anche in questo caso manca una indicazione più precisa, ma si tratta di: J. Burckardt. Die kultur der Renaissancein Italien, Seemann, Leipzig 1869 (ed infatti l'autore dice che si tratta di un'opera di "trent'anni fa").

82 Questa affermazione è confermata da quanto scrive G. Carotti. Vicende del Duomo di Milano e della sua facciata, in Archivio storico dell'Arte, anno II, 1889, fascicoli III-IV e VII, anche in estratto, Società Tipografico-Editrice Laziale, Roma 1889. 
sostanzialmente affermata nel bando di concorso di seconda fase, che il fianco debba corrispondere alla fronte. ${ }^{83}$ Lo stesso progetto vincitore gli appare il frutto di un cedimento alla freddezza della ragione, molto migliore il primo presentato dallo stesso Brentano. Egli in effetti sostiene preferibili soluzioni che muovano decisamente verso l'orizzontalità o che accentuino veramente la verticalità con la progettazione di due torri. I suoi favori quindi vanno verso il primo progetto di Brentano, le proposte di Cesa Bianchi e di Locati. Egli vorrebbe anche, per mantenere lo spirito del monumento, un progetto non soltanto orizzontale ma anche con proporzioni rinascimentali in vesti gotiche.

E' da notare che egli comunque vorrebbe un volo di fantasia, una grande audacia, ricordando a questo proposito la risposta data da Paolo Calco a Mignot quando questi sosteneva una propria soluzione architettonica con la frase che esistevano in Francia molti archi come quelli da lui proposti, e cioè che il Duomo non aveva nulla a che vedere con cose vecchie, ma è cosa del tutta nuova. Una novità che egli non vede nei progetti presentati, non per colpa del vincitore ma per tre errori dovuti in parte all'ambiente in parte alla struttura del bando: ritenere il Duomo lombardo; seguire la scuola della logica della ragione desunta da "quel Viollet-Le-Duc" che ha molti seguaci a Milano, che vorrebbe che l'architetto divenisse, per così dire, servo dell'architettura, quasi astrazione ideale e non oggetto reale, frutto della volontà di chi la costruisce; il programma del concorso e la commissione di giudizio, che hanno dimenticato il carattere duplice del monumento scegliendo una sola via e provocando così inestricabili contraddizioni. ${ }^{84}$ Geymüller che vorrebbe un intervento di grande audacia, non necessariamente legato alle preesistenze, sia organicamente che stilisticamente, è anche l'unico che si pone il problema della liceità della ricostruzione, che risolve in modo positivo.

Le osservazioni che rilevano la grande qualità delle parti architettoniche del Pellegrini sono numerose, e la testimonianza più significa-

83 Anche in questo caso vengono indicati casi concreti relativi ad architetture gotiche e lombarde (Nôtre Dame, Cattedrale di Strasburgo, Certosa di Pavia, ma soprattutto il grande esempio dell'ospedale degli Innocenti di Firenze, dove Brunelleschi usa stili diversi all'esterno ed all'interno. Da ciò una particolare attenzione al progetto presentato da Ciaghin al primo grado di concorso, molto sviluppato in senso orizzontale.

84 I progetti che a suo parere hanno meglio superato le contraddizioni sono quelli di Cesa Bianchi, Ferrario presentati nel concorso, e quello esposto nel suo scritto da Aristide Nardini Despotti Mospignotti, vedi nota 45. 
tiva resta il progetto di Beltrami per la costruzione di un campanile, in asse con la galleria e dalla parte opposta della piazza.

L'attenzione verso il valore della preesistenza si accentua verso la fine del secolo e nei primi anni del successivo: emergono tendenze sempre più favorevoli ad un intervento che non alteri sostanzialmente la fronte del Duomo. Si possono rilevare due atteggiamenti. Il primo pone l'accento sul grado di affezione verso il Duomo da parte dei milanesi, le cui forme fanno oramai parte della coscienza comune: i cittadini sono abituati a vederlo così e difficilmente si dimostrano favorevoli ad un mutamento. Si tratta peraltro di un sentimento che certamente è presente in chi ha scritto, impossibile da accertare per quanto riguarda la popolazione. Altre considerazioni si riferiscono invece ad un principio di restauro, alla consapevolezza che è contraddittorio affermare che le stratificazioni storiche sui monumenti siano da conservare se di rilevanza storica o artistica, riconoscere la bellezza degli interventi pellegriniani e proporne la rimozione per raggiungere un'unità di stile che è stata esclusa dagli obbiettivi del restauro.$^{85}$ In molti casi sembra mutare l'oggetto della disputa: non si parla più di nuova fronte, ma della sua riforma. ${ }^{86}$

La prima organica esposizione di questo nuovo atteggiamento si trova in Cesare $\mathrm{Nava}^{87}{ }^{87}$ che afferma non doversi scompaginare la parte inferiore del Duomo neppure per usarne gli elementi in altri edifici,

85 Il riferimento costante, implicito o esplicito, è alle tesi enunciate da Camillo Boito nel 1883, vedi nota 32.

86 Va ricordato anche l'aspetto economico della questione: il completamento ottocentesco, iniziato nel 1806 e terminato nel 1813, era costato circa 2.400 .000 lire che corrispondevano a circa 4.000.000 della fine degli anni ' 80 del XIX secolo; questo fa pensare che il rifacimento della fronte, nei progetti che richiedevano minore sacrificio della preesistenza, come quelli di Brentano e di Beltrami, potessero costare non meno di 6 milioni di lire, a fronte delle 820.000 lasciate da De Togni e delle 120.000 di assegno annuo governativo per le spese di fabbriceria. D'altronde nel bando di concorso per la nuova fronte era stato esplicitamente escluso che si facessero preventivi, nella presunzione che le risorse per una così grande impresa non sarebbero mancate. La relativa mancanza di denaro, e la necessità di non perdere il lascito, decretando la fine dell'opera per la fronte, sono i motivi principali della riforma che conduce alla soppressione del coronamento di Amati, deliberato definitivamente nel 1909; si veda la pubblicazione, a cura della fabbriceria, Per la facciata del Duomo di Milano- Relazione all'on. Ministro della Pubblica Istruzione, Sonzogno, Milano 1909.

87 C. Nava, La facciata del Duomo, dispareri e proposte, Tipografia Pulzato e Giani, Milano 1901. (Conferenza tenuta per incarico dell'associazione "Religione e Patria” per gli interessi pubblici). 
contrario quindi alla proposta di Beltrami di utilizzarli in un nuovo campanile, perché la loro qualità consisteva non soltanto nella bellezza dei singoli elementi, ma anche nell'assieme, per la libertà di linee, per l'intreccio felicissimo tra decorazione ed architettura. Egli tuttavia, a conferma della diffidenza di Boito per ragionamenti troppo stretti, per salvare quell'integrità che legge nel contesto e salvare l'unità stilistica della facciata, propone il trasporto della parte inferiore della fronte per chiudere la corte del palazzo reale. ${ }^{88}$

Carlo Romussi, che aveva dedicato un volume al Duomo ed alla sua storia ${ }^{89}$ in cui dichiara decisamente il suo favore per il progetto di Luca Beltrami,${ }^{90}$ più di altri si sofferma sulle difficoltà di realizzazione del progetto di Brentano, anche dopo la decisione di darne esecuzione parziale, limitata alla parte centrale più alta della fronte. La porta principale, che era stata oggetto delle più alte lodi da parte della commissione di concorso suscita molte perplessità. "Ed ecco insensibilmente formarsi nella cittadinanza un'opinione contraria al cambiamento della facciata", con azioni promosse dai circoli artistici che avevano il consenso popolare; egli vi vede anche il superamento nel restauro delle tesi di Viollett-Le-Duc. ${ }^{91}$ Romussi cita anche precedenti a

88 A questa ipotesi Giulio Carotti, nello studio citato a nota 24, obbietta che così lo spazio attorno al Duomo sarebbe stato troppo ristretto.

89 C. Romussi, Intorno alla facciata del Duomo di Milano- Considerazioni e proposte, Sonzogno, Milano 1904.

90 La posizione è particolarmente significativa perché Carlo Romussi era direttore del quotidiano Il Secolo, che si collocava politicamente su di un versante che, con linguaggio di oggi, si potrebbe definire di centro sinistra, in continua polemica con il Corriere della Sera e soprattutto con la Perseveranza; in questa veste ben raramente in accordo con Luca Beltrami.

91 Dice Romussi: "Cominciarono gli artisti... oppure fu il popolo che non voleva fosse sottratta a' suoi occhi la facciata del monumento che è il simbolo e l'orgoglio della città ? [...] Si era cambiata l'aria: altre idealità avevano sostituito quelle diffuse dal Viollet-Le-Duc. La voce degli artisti, espressione della nuova tendenza, diceva doversi conservare ogni manifestazione sincera di arte, perché testimonianza di un tipo e di un tempo, e in questo caso trattarsi di un'opera d'arte che sarebbe stato sacrilegio abbattere: e la voce popolare chiedeva si rispettasse il suo duomo nella fisionomia che tutti erano abituati a vedere." Op. cit. cfr. nota 70, pp.56-57. I riferimenti concreti "agli artisti" potrebbero essere i voti della Commissione Conservatrice dei Monumenti, dell'Accademia di Brera, dell'Assemblea degli artisti lombardi, tutti favorevoli alla conservazione della facciata del Duomo (citati da Carotti, cfr. nota 82), ai quali si aggiunge però l'azione della commissione per la facciata del Duomo, formata nel 1902 e di cui egli stesso faceva parte, che sospende i lavori in corso. 
questa posizione, che vanno dal rimpianto per la perdita delle parti antiche espresse da Brentano, Guidini, Nava e Beltrami, ${ }^{92}$ di quest'ultimo anche il vero e proprio dubbio espresso sulla correttezza dell'idea di sopprimere la nuova fronte. Egli infatti aveva affermato nel testo che prepara la partecipazione al concorso, che occorreva pregiudizialmente “...esaminare e ponderare con tutta serietà, se realmente il progetto che si vorrà tradurre in atto sia tale da giustificare la scomposizione dell'attuale facciata".93

92 Carlo Romussi, op. cit. a nota 71, senza dare indicazioni bibliografiche, cita parole di Giuseppe Brentano che avverte come il progetto della nuova fronte imponga mentalmente la soppressione dell'opera di grandi artisti del passato, che, o per eccessivo amore verso una sola forma d'arte, o per fredda imitazione di stili, o per non aver saputo immedesimarsi nel concetto dei primi architetti, hanno fatto opera discordante; molte parti scompariranno senza rimpianti, ma non le porte pellegriniane di cui tesse gli elogi, sia per la qualità dei singoli membri architettonici, sia per il partito orizzontale che inseriscono nel Duomo (cita in effetti da G. Brentano. Per la nuova facciata del Duomo di Milano, Ricordi, Milano 1888). Egli scrive, romanticamente, che anche il più freddo degli uomini sarebbe stato percorso da un brivido "al primo colpo di martello che intaccasse l'opera dei nostri padri" . Il passo è ripreso da Beltrami che richiamandosi a quelle parole di Brentano dichiara di aver provato quel brivido quando, nella Pasqua del 1900, " per la navata del tempio cominciò ad echeggiare il lavoro dei ponti di servizio, e sul volto di chi impartiva gli ordini, cercai indarno la serena convinzione dell'opera avviata". Va ricordato lo scritto A. Manfredini. La facciata del Duomo di Milano, in Il Monitore tecnico, anno V, n. 32, Milano 1899, anche in estratto autonomo, stessa data, che presenta una storia della questione della facciata del Duomo sintetica ma ricca di informazioni precise; egli riprende le parole di Brentano qui citate per contrastarle decisamente (p. 16 dell'estratto) Romussi, anche in questo caso senza indicazione bibliografica, cita da A. Guidini. La facciata del Duomo attraverso $i$ secoli e gli attuali concors $i$, in Illustrazione Italiana, Treves, Milano 11 settembre 1887, numero speciale intitolato Il concorso mondiale per la Nuova Facciata del Duomo di Milano., dove l'autore dichiara che le parti del Pellegrini sono documenti storici da conservare, benché scriva anche che l'errore del completamento postnapoleonico sia consistito nell'aver mantenuto il "falsato organismo", di aver innestato due stili, di aver operato con cattive tecniche, pessimi materiali, mal lavorati, aggiungendo inoltre che ulteriore gravissimo errore era stata l'eliminazione della piattaforma gradinata di accesso. L. Beltrami. Per la facciata del Duomo di Milano, ..., Milano 1887, aveva definito "il disperdimento della parte inferiore... un vero atto di vandalismo" osservando che "... già si manifestano gli indizî di una corrente di idee, secondo la quale tali frammenti, non solo debbono essere lasciati per l'intrinseco loro valore artistico, ma debbono essere lasciati in posto, essendo un elemento interessante per la storia dell'arte."

93 Carlo Romussi, op. cit. qui sopra, pp. 58-59, che non dà riferimenti ma cita da Luca Beltrami, Per la facciata del Duomo di Milano, ..., Milano 1887. 
Giulio Carotti, a conclusione del suo studio del $1889,{ }^{94}$ affronta il tema, ${ }^{95}$ che svilupperà più a pieno durante le discussioni per il nuovo coronamento che chiuderanno la questione della facciata, nel $1909^{96}$ con l'abbandono dell'ipotesi del suo rifacimento integrale, per la più modesta sostituzione del coronamento di epoca napoleonica.

94 G. Carotti, op.cit, vedi nota 24.

95 "Di un altro argomento meriterebbe ancora tener breve cenno .... Questa trasformazione trae seco la scomparsa dalla fronte delle cinque porte e di due delle finestre inferiori laterali, le quali sono oramai già creazioni antiche, creazioni in forte dissonanza con lo stile del duomo, è vero, ma in se stesse grandiose e bellissime opere d'arte, alle quali son legati i nomi dei più chiari artisti, architetti, pittori e scultori che operarono pel Duomo nel XIV e XVII secolo...".

96 Nel 1909, falliti anche i tentativi di una riforma della fronte secondo un progetto elaborato da una commissione presieduta da Boito, si decide per il semplice rifacimento del coronamento, che aveva dato anche segni di qualche cedimento statico. Alla decisione si oppongono due commissari. Giuseppe Bagatti Valsecchi, che considera un obbrobrio il coronamento in opera, ma che ne desidera il consolidamento perché comprende che quell'atto amministrativo segna l'abbandono del progetto di fare la nuova fronte. Giulio Carotti invece, in una articolata relazione di minoranza, dichiara che lo studio dei monumenti del passato lo ha portato alla convinzione che essi devono essere riparati, consolidati, rifatti eventualmente nelle parti pericolanti, ma mai modificati nello stile, nell'organismo e nelle forme, neppure negli elementi secondari o modificati nei tempi successivi, sebbene discordanti o deficienti di pregio, tanto più nei monumenti di alta rilevanza storica come il Duomo. Egli cita a questo proposito numerose prese di posizione di organismi ufficiali, nazionali e milanesi, dal 1904 al 1907. Cfr. Per la facciata del Duomo di Milano- Relazione all'on. Ministro della Pubblica Istruzione, Sonzogno, Milano 1909, pp. 21-23. 
